\title{
A Reaction Diffusion Model to Describe the Toxin Effect on the Fish-Plankton Population
}

\author{
Wendkouni Ouedraogo, Hamidou Ouedraogo, and Boureima Sangaré $\mathbb{D}$ \\ Department of Mathematics and Informatics, UFR/ST, Université Nazi Boni, 01 BP 1091, Bobo-Dioulasso 01, Burkina Faso \\ Correspondence should be addressed to Boureima Sangaré; mazou1979@yahoo.fr
}

Received 10 December 2017; Accepted 5 March 2018; Published 10 May 2018

Academic Editor: Kenneth Karlsen

Copyright (c) 2018 Wendkouni Ouedraogo et al. This is an open access article distributed under the Creative Commons Attribution License, which permits unrestricted use, distribution, and reproduction in any medium, provided the original work is properly cited.

This paper is aimed at the mathematical formulation, the analysis, and the numerical simulation of a prey-competitor-predator model by taking into account the toxin produced by the phytoplankton species. The mathematical study of the model leads us to have an idea on the existence of solution, the existence of equilibria, and the stability of the stationary equilibria. These results are obtained through the principle of comparison. Finally, the numerical simulations allowed us to establish a threshold of release of the toxin, above which we talk about the phytoplankton blooms.

\section{Introduction}

Understanding the functioning of an ecosystem is a major issue for resource and environmental management. However, this goal remains difficult to attain due to the complexity of natural systems, especially in the aquatic environment, where many processes of all types interact with living organisms [1-3]. Plankton is the basis of all aquatic food chains and phytoplankton in particular occupies the first trophic level and the fluctuations in its abundance determine the production of marine environment. Rapid increase and almost equally rapid decrease separated by periods are the common features of plankton populations. In a broad sense, planktonic blooms can be divided into two types: "spring blooms" and "red tides." Spring blooms occur seasonally for the changes in temperature or nutrient availability which are connected with seasonal changes in thermocline depth, strength, and consequent mixing. Red tides are localized outbreaks and occur due to high water temperature [4-7]. Toxic substances produced by phytoplankton species reduce the growth of zooplankton by decreasing grazing pressure and this is one of the important common phenomena in plankton ecology $[2,6,8,9]$.
Within the broad perspective drawn above, the present paper explores and compares the coupled dynamics of the phytoplankton and the zooplankton in a number of mathematical models. The system phytoplankton-zooplankton has attracted considerable attention from various fields of research $[3,8,10,11]$. It is an important issue in mathematical ecology. The literature abounds in models focusing on various aspects of the problem. Recently, the attention has been focused on the role of the space in explaining heterogeneity and the distribution of the species and the influence of the spatial structure on their abundance $[2,6,8]$. However, the very question of the interactions between phytoplankton, zooplankton, and fish depending on space is far from being fully elucidated. As part of our work, we will highlight a threshold value of the toxin released by phytoplankton, below which the effect of the toxin influences less the dynamics of the zooplankton-fish system. To our knowledge, this has never been addressed in the literature. The proposed model consists of three interactive components, zooplankton, herbivorous fish, and toxin-producing phytoplankton, which reduce the growth of fish and zooplankton population. The model studied here is of the reaction-diffusion type, describing the dynamics of the phytoplankton-zooplankton-fish 


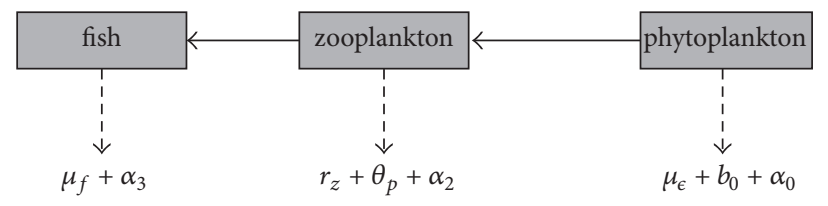

FIGURE 1: Typical ecological situation presented by food-chain model.

system in the sense of the work of Courchamp et al. [12].

The paper is organized as follows. As far as Section 3 is concerned, we will establish mathematical results such as the existence of a solution, stability of equilibria, and persistence, relating to the constructed model in the Section 2. Section 4 will be devoted to numerical experiments to illustrate the mathematical results. Finally, Section 5 is devoted to the conclusion and perspectives.

\section{Mathematical Model}

In this section, we propose a model to describe the dynamics of the phytoplankton-zooplankton-fish system in the presence of toxin. We begin our modeling by a general model describing the dynamics of the prey-competitor-predator system, based on the equations with ordinary derivatives. And then we transform this model into a model of reactiondiffusion type while remaining in the logic of the works of Courchamp et al. [12] and Bendahmane [13]. It should be noted that the aim is to take into account the effect of the toxin on the fish population through that of zooplankton.

2.1. Original Model Formulation. If we designate by $P$ the density of the prey, by $Z$ the density of the competitor, and by $F$ that of the predator, according to [12], the general model is written as follows:

$$
\begin{aligned}
& \frac{d P}{d T}=\phi_{2}(P)-g_{5}(P, Z) P-g_{6}(P, Z) Z \\
& \frac{d Z}{d T}=g_{7}(P, Z) Z-g_{8}(P, Z, F) Z-g_{9}(P, Z, F) F \\
& \frac{d F}{d T}=g_{10}(Z, F) F-g_{11}(Z, F) F,
\end{aligned}
$$

where

(i) $\phi_{2}, g_{5}, g_{6}, g_{7}, g_{8}, g_{9}, g_{10}$, and $g_{11}$ are positive $C^{\infty}$ functions,

(ii) $\phi_{2}(P)$ is the growth function of the prey,

(iii) $g_{5}(P, Z)$ is the function that measures the mortality due to the competition between the same individuals of the prey,

(iv) $g_{6}(P, Z)$ is the function that measures the mortality of the prey due to the competitor's consumption,

(v) $g_{7}(P, Z)$ is the competitor's growth function,

(vi) $g_{8}(P, Z, F)$ is the function that measures the mortality of the competitor from the release of the substance produced by the prey, (vii) $g_{9}(P, Z, F)$ is the function that measures the mortality of the competitor from the consumption of the predator,

(viii) $g_{10}(Z, F)$ is the growth function of the predator,

(ix) $g_{11}(Z, F)$ is the function that measures the external mortality of the predator population.

We continue our modeling by fixing the expressions of the functions intervening in model (1). The dynamics of the system are represented by Figure 1 .

According to Figure 1, for any time $T>0$, the dynamics of the phytoplankton (prey)-zooplankton (competitor)-fish (predator) system is governed by the following ODE system:

$$
\begin{aligned}
& \frac{d P}{d T}=r_{p} P-\mu_{\epsilon} P-b_{0} P^{2}-\frac{\alpha_{0} Z}{P+\gamma_{0}} P, \quad P(0)=P_{0} \geq 0, \\
& \frac{d Z}{d T}=-r_{z} Z-\theta_{p} Z+\frac{\alpha_{1} P}{P+\gamma_{0}} Z-\frac{\alpha_{2} F}{Z+\gamma_{2}} Z, \\
& \qquad Z(0)=Z_{0} \geq 0, \\
& \frac{d F}{d T}=r_{f} F-\mu_{f} F-\frac{\alpha_{3} F^{2}}{Z+\gamma_{3}}, \quad F(0)=F_{0} \geq 0,
\end{aligned}
$$

where

(i) $r_{p}$ and $\mu_{\epsilon}$ denote, respectively, the phytoplankton growth rate and the phytoplankton external mortality rate,

(ii) $b_{0}$ and $\alpha_{0}$ denote, respectively, the mortality due to competition between the individuals of the phytoplankton population and the specific predation rate of the zooplankton on the phytoplankton population,

(iii) $\gamma_{0}$ is the half-saturation constant of zooplankton predation on the phytoplankton,

(iv) $r_{z}$ denotes the external mortality rate of zooplankton,

(v) $\theta_{p}$ denotes the rate of toxin production per phytoplankton species,

(vi) $\alpha_{1}$ and $\alpha_{2}$ represent, respectively, the maximum value that the consumption rate of the phytoplankton by the zooplankton can reach and the maximum value that the reduction rate by the zooplankton can reach,

(vii) $\gamma_{2}$ is the value of zooplankton for which the elimination rate by the individual zooplankton becomes $\alpha_{2} / 2$,

(viii) $r_{f}$ and $\mu_{f}$ represent, respectively, the growth rate and the external mortality rate of the fish,

(ix) $\alpha_{3}$ is the maximum value that the rate of reduction by the fish can reach, 
(x) $\gamma_{3}$ represents the residual loss caused by high rarity of the zooplankton of individuals of the fish population.

2.2. A Spatially Structured Model. Considering the relationship between the climate and the diffusion of species and the fact of the existence of diffusion in population, system (1) is developed into a spatial system with diffusion. We expect to explore the effect of climate change on plankton population by studying the spatial dynamics of the diffusion system. We will introduce the concept of spatial structure in the model; that is to say, the population's densities depend now on the time and space. Diffusion models are a simple and reasonable choice for modeling dispersion of populations on a spatial domain $[14,15]$. Indeed, let $\delta_{1}(x), \delta_{2}(x)$, and $\delta_{3}(x)$ be, respectively, the diffusivity coefficients of $P, Z$, and $F$. Based on the work established in [12], the structured model associated with model (1) can be modeled for $x \in \Omega$ and $T>0$ as follows:

$$
\begin{aligned}
& \partial_{T} P-\operatorname{div}\left(\delta_{1}(x) \nabla P\right) \\
& \quad=\phi_{2}(P)-g_{5}(P, Z) P-g_{6}(P, Z) Z, \\
& \partial_{T} Z-\operatorname{div}\left(\delta_{2}(x) \nabla Z\right) \\
& \quad=g_{7}(P, Z) Z-g_{8}(P, Z, F) Z-g_{9}(P, Z, F) F, \\
& \partial_{T} F-\operatorname{div}\left(\delta_{3}(x) \nabla F\right)=g_{10}(Z, F) F-g_{11}(Z, F) F,
\end{aligned}
$$

where $\Omega \subset \mathbb{R}^{n}(n \geq 2)$ is the spatial domain in which species occur and zero-flux boundary condition.

$$
\delta_{i}(x) \nabla Q(x, t) \cdot \nu(x)=0,
$$

$$
i=1,2,3, x \in \partial \Omega, t>0,
$$

for $Q=P, Z, F$, where $\nu$ is the unit normal vector to $\Omega$ along $\partial \Omega$ and nonnegative initial conditions

$$
Q(x, 0)=Q_{0}(x)>0, \quad Q=P, Z, F, x \in \Omega .
$$

So, model (2) can be written by the following system:

$$
\begin{aligned}
\partial_{T} P & \\
= & \left(r_{p}(x)-\mu_{\epsilon}(x)-b_{0}(x) P-\frac{\alpha_{0}(x) Z}{P+\gamma_{0}(x)}\right) P \\
& +\operatorname{div}\left(\delta_{1}(x) \nabla P\right), \\
\partial_{T} Z & \\
= & \left(-r_{z}(x)-\theta_{p}(x)+\frac{\alpha_{1}(x) P}{P+\gamma_{0}(x)}-\frac{\alpha_{2}(x) F}{Z+\gamma_{2}(x)}\right) Z \\
& +\operatorname{div}\left(\delta_{2}(x) \nabla Z\right), \\
\partial_{T} F \quad & \quad\left(r_{f}(x)-\mu_{f}(x)-\frac{\alpha_{3}(x) F}{Z+\gamma_{3}(x)}\right) F \\
& +\operatorname{div}\left(\delta_{3}(x) \nabla F\right) .
\end{aligned}
$$

We make the following assumptions:
$\left(\mathrm{H}_{a}\right)$ All demographic parameters of system (6) are positive constants.

$\left(\mathrm{H}_{b}\right)$ The diffusivity coefficients of system (6) are independent of $x$.

Let us consider $\delta_{1}(x)=\delta_{1}, \delta_{2}(x)=\delta_{2}$, and $\delta_{3}(x)=\delta_{3}$ in the remaining of our work.

Taking into account hypotheses $\left(\mathrm{H}_{a}\right)$ and $\left(\mathrm{H}_{b}\right)$, model (6) becomes

$$
\begin{gathered}
\partial_{T} P=\left(r_{p}-\mu_{\epsilon}-b_{0} P-\frac{\alpha_{0} Z}{P+\gamma_{0}}\right) P+\delta_{1} \Delta P, \\
P(x, 0)=P_{0}(x)>0, \\
\partial_{T} Z=\left(-r_{z}-\theta_{p}+\frac{\alpha_{1} P}{P+\gamma_{0}}-\frac{\alpha_{2} F}{Z+\gamma_{2}}\right) Z+\delta_{2} \Delta Z, \\
Z(x, 0)=Z_{0}(x)>0, \\
\partial_{T} F=\left(r_{f}-\mu_{f}-\frac{\alpha_{3} F}{Z+\gamma_{3}}\right) F+\delta_{3} \Delta F, \\
F(x, 0)=F_{0}(x)>0 .
\end{gathered}
$$

Remark 1. The model presented here is a model of predator prey system with a self-diffusion but it is also possible to extend these types of system to systems with a cross-diffusion in the same logic of the works of Andreianov et al. [16, 17].

\section{Mathematical Results}

The mathematical results are based on the works in $[3,16,18-$ 20].

3.1. Reduction of Model Parameters. To simplify the writing, we will make the following changes of variables:

$$
\begin{aligned}
a_{0} & =r_{p}-\mu_{\epsilon}, \\
a_{1} & =r_{z}+\theta_{p}, \\
c_{3} & =r_{f}-\mu_{f}, \\
U_{1}(t) & =\frac{b_{0}}{a_{0}} P(T), \\
U_{2}(t) & =\frac{b_{0} \alpha_{0}}{a_{0}^{2}} Z(T), \\
t & =a_{0} T, \\
p & =\frac{c_{3}}{a_{0}}, \\
q & =\frac{\alpha_{3}}{\alpha_{2}}, \\
y & =Y \sqrt{a_{0}}, \\
a & =\frac{b_{0} \gamma_{0}}{a_{0}},
\end{aligned}
$$




$$
\begin{aligned}
b & =\frac{a_{1}}{a_{0}}, \\
x & =X \sqrt{a_{0}}, \\
r & =\frac{b_{0} \alpha_{0} \gamma_{3}}{a_{0}^{2}}, \\
d & =\frac{b_{0} \alpha_{0} \gamma_{0}}{a_{0}^{2}}, \\
\epsilon_{3} & =\frac{a_{0}^{3}}{b_{0} \alpha_{0} \alpha_{1}} \delta_{3}, \\
\epsilon_{1} & =\frac{a_{0}}{b_{0}} \delta_{1}, \\
\epsilon_{2} & =\frac{a_{0}^{2}}{b_{0} \alpha_{0}} \delta_{2}, \\
z & =Z \sqrt{a_{0}}, \\
c & =\frac{\alpha_{1}}{a_{0}}, \\
U_{3}(t) & =\frac{b_{0} \alpha_{0} \alpha_{1}}{a_{0}^{3}} F(T) .
\end{aligned}
$$

Thus, we obtain, respectively, systems (2) and (7) of the following forms:

$$
\begin{aligned}
& \frac{d E_{1}}{d t}=\left(1-E_{1}+\frac{E_{2}}{E_{1}+a}\right) E_{1}, \\
& \frac{d E_{2}}{d t}=\left(-b+\frac{c E_{1}}{E_{1}+a}-\frac{E_{3}}{E_{2}+d}\right) E_{2}, \\
& \frac{d E_{3}}{d t}=\left(p-\frac{q E_{3}}{E_{2}+r}\right) E_{3}, \\
& \partial_{t} U_{1}=\left(1-U_{1}+\frac{U_{2}}{U_{1}+a}\right) U_{1}+\epsilon_{1} \Delta U_{1}, \\
& \partial_{t} U_{2}=\left(-b+\frac{c U_{1}}{U_{1}+a}-\frac{U_{3}}{U_{2}+d}\right) U_{2}+\epsilon_{2} \Delta U_{2}, \\
& \partial_{t} U_{3}=\left(p-\frac{q U_{3}}{U_{2}+r}\right) U_{3}+\epsilon_{3} \Delta U_{3} .
\end{aligned}
$$

3.2. Existence and Boundedness of Solution. Before stating the boundedness of the solution, we give the following theorem concerning the existence and uniqueness of the solution of system (7).

Theorem 2 (see $[16,21-23])$. System (7) has a unique local solution $\left(U_{1}(\cdot, t), U_{2}(\cdot, t), U_{3}(\cdot, t)\right)$ under the condition $0 \leq t<$ $T_{\max }$, where $T_{\max }$ depends on nonnegative initial data $U_{01}(x)$, $U_{02}(x)$, and $U_{03}(x)$.

The following theorem ensures the boundedness of the solution.
Theorem 3. The set $\mathscr{D}=[0,1] \times[0, c+c / 4 b] \times[0,1 / \alpha+1 / 4 \alpha b+$ $M / \alpha b]$ attracts all global solutions of system (7) initiating in the positive orthant, where $\alpha=4 b /(b+p)^{2}(4 b c+c+4 b r)$.

Proof. By noticing that $U_{1} \leq E_{1}, U_{2} \leq E_{2}$, and $U_{3} \leq E_{3}$ and from $[10,19]$, we have $\overline{\lim _{t \rightarrow+\infty}} E_{1}(t) \leq 1$ and $\varlimsup_{\lim _{t \rightarrow+\infty}}\left(E_{1}(t)+\right.$ $\left.(1 / c) E_{2}(t)\right) \leq 1+1 / 4 b$. Finally, we get that

$$
\varlimsup_{t \rightarrow+\infty}\left(E_{1}(t)+\frac{1}{c} E_{2}(t)+\alpha E_{3}(t)\right) \leq 1+\frac{1}{4 b}+\frac{M}{\alpha b} .
$$

The following theorem gives the existence of global solution.

Theorem 4 (see [21-23]). System (10) admits a global solution $\left(U_{1}(\cdot, t), U_{2}(\cdot, t), U_{3}(\cdot, t)\right)$ for any $t>0$ and for any regular initial positive functions $U_{01}(x) \leq 1, U_{02}(x)$, and $U_{03}(x)$.

Proof. In fact,

(i) on one hand, we have $U_{1}(x, t) \geq 0, U_{2}(x, t) \geq 0$, and $U_{3}(x, t) \geq 0$ because 0 is the subsolution of each equation of system (10);

(ii) on the other hand, $U_{1}$ satisfies the following problem:

$$
\begin{aligned}
\partial_{t} U_{1} & \leq U_{1}\left(1-U_{1}\right)+\epsilon_{1} \Delta U_{1} \quad \text { on } \Omega \times[0,+\infty[, \\
\partial_{\nu} U_{1} & =0, \quad \text { on } \partial \Omega \times[0,+\infty[, \\
U_{1}(x, 0) & =U_{01}(x) \leq U_{01} \equiv \max _{\bar{\Omega}} U_{01}(x), \quad x \in \Omega .
\end{aligned}
$$

According to the principle of comparison, we have $U_{1}(x, t) \leq$ $U(t) \leq 1$, where $U(t)=U_{01} e^{t} /\left(1-U_{01}+e^{t}\right)$ is the solution of the system

$$
\begin{aligned}
\frac{\partial U}{\partial t} & =U(1-U), \\
U(0) & =U_{01} \leq 1 .
\end{aligned}
$$

In the same order, $U_{2}$ satisfies $\partial U_{2} / \partial t=c U_{1} U_{2} /\left(U_{1}+a\right)-$ $b U_{2}-U_{2} U_{3} /\left(U_{2}+d\right)+\epsilon_{2} \Delta U_{2}$.

According to the principle of comparison, we have $(1 / c)\left(\partial U_{2} / \partial t\right) \leq(1 / c)\left(d E_{2} / d t\right)$, where $E_{2}$ is the solution of the second equation of system (2) with initial condition $E_{2}(0)$ $=\max _{\bar{\Omega}} U_{02}(x)$.

According to $[10,19]$, we have $(1 / c)\left(\partial U_{2} / \partial t\right) \leq$ $(1 / c)\left(d E_{2} / d t\right)+d E_{1} / d t$. Let us denote $\sigma=(1 / c) E_{2}+E_{1}$; then $(1 / c)\left(\partial U_{2} / \partial t\right) \leq d \sigma / d t \leq 1 / 4+b-b \sigma$. Therefore, we have $U_{2}(x, t) \leq c+c / 4 b, 0 \leq t<T$.

From the third equation of system (10), we find $\alpha\left(\partial U_{3} /\right.$ $\partial t)=\alpha p U_{3}-\alpha q U_{3}^{2} /\left(U_{2}+r\right)+\alpha \epsilon_{3} \Delta U_{3}$. From the comparison principle, we can deduce that $\alpha\left(\partial U_{3} / \partial t\right) \leq \alpha\left(d E_{3} / d t\right)$, where $E_{3}$ is the solution of the third equation of system (2) with initial condition $E_{3}(0)=\max _{\bar{\Omega}} U_{3}(x)$. Therefore,

$$
\begin{aligned}
\alpha \frac{\partial U_{3}}{\partial t} & \leq \alpha \frac{d E_{3}}{d t}+\frac{1}{c} \frac{d E_{2}}{d t}+\frac{d E_{1}}{d t} \\
& \leq E_{1}\left(1-E_{1}\right)-\frac{b}{c} E_{2}-\frac{1}{c} \frac{E_{2} E_{3}}{E_{2}+d}
\end{aligned}
$$




$$
\begin{aligned}
& +\alpha\left(p-\frac{q E_{3}}{E_{2}+r}\right) E_{3} \\
\leq & \frac{1}{4}+b-\alpha b U_{3}+\alpha b E_{3}+\alpha\left(p-\frac{q E_{3}}{E_{2}+r}\right) E_{3} .
\end{aligned}
$$

Then,

$$
\alpha \frac{\partial U_{3}}{\partial t}+\alpha b U_{3} \leq \frac{1}{4}+b+M, \quad M=\frac{1}{4 q} .
$$

And thus,

$$
U_{3}(x, t) \leq \frac{1}{\alpha}+\frac{1}{4 \alpha b}+\frac{M}{\alpha b}, \quad 0 \leq t<T .
$$

3.3. Analysis of Stationary Solutions. The positive equilibria are the solutions $\left(U_{1}(x), U_{2}(x)\right.$, and $\left.U_{3}(x)\right)$ of system (7), where $U_{1}(x) \geq 0, U_{2}(x) \geq 0$, and $U_{3}(x) \geq 0$. Suppose that there exist two positive constants $m_{1}<1$ and $m_{2}$ such that $U_{1}(x) \geq m_{1}>0, U_{2}(x) \geq m_{2}>0$, and $U_{3}(x)>0$. The equilibrium of system (7) satisfies

$$
\begin{aligned}
-\epsilon_{1} \Delta U_{1} & =U_{1}\left(1-U_{1}\right)-\frac{U_{1} U_{2}}{U_{1}+a}=F^{*}\left(U_{1}, U_{2}, U_{3}\right), \\
-\epsilon_{2} \Delta U_{2} & =\frac{c U_{1} U_{2}}{U_{1}+a}-b U_{2}-\frac{U_{2} U_{3}}{U_{2}+d}=G^{*}\left(U_{1}, U_{2}, U_{3}\right), \\
-\epsilon_{3} \Delta U_{3} & =p U_{3}-\frac{q U_{3}^{2}}{U_{2}+r}=H^{*}\left(U_{1}, U_{2}, U_{3}\right), \\
\frac{\partial U_{1}}{\partial v} & =\frac{\partial U_{2}}{\partial \nu}=\frac{\partial U_{3}}{\partial v}=0 \quad \text { on } \partial \Omega .
\end{aligned}
$$

We make the following assumptions:

$$
\begin{aligned}
& \left(H_{1}\right) \quad c+\frac{c}{4 b} \leq \frac{m_{1}+a}{m_{1}}\left[\frac{m_{2}}{1+a}+m_{1}\left(1-m_{1}\right)\right] \\
& \left(H_{2}\right) \quad b \geq \frac{c}{1+a}, \\
& \quad \frac{1}{\alpha}+\frac{c}{4 b \alpha}+\frac{M}{\alpha} \leq \frac{c m_{1}}{m_{1}+a}-b-\frac{\overline{U_{3}}}{m_{2}+d} \\
& \left(H_{3}\right) \quad \frac{p}{q}\left(m_{2}+r\right) \leq \frac{1}{\alpha}+\frac{c}{4 b \alpha}+\frac{M}{\alpha} .
\end{aligned}
$$

Theorem 5. Let assumptions $\left(H_{a}\right),\left(H_{1}\right),\left(H_{2}\right)$, and $\left(H_{3}\right)$ hold; system (17) has at least one positive solution.

Proof. We will construct a pair of subsolution and oversolution of problem (17):

$$
\left(\bar{U}_{1}(x), \bar{U}_{2}(x), \bar{U}_{3}(x)\right)>\left(\underline{U_{1}}(x), \underline{U_{2}}(x), \underline{U_{3}}(x)\right),
$$

which verifies

$$
\begin{gathered}
-\epsilon_{1} \Delta \bar{U}_{1}-\bar{U}_{1}\left(1-\bar{U}_{1}\right)-\frac{\bar{U}_{1} \bar{U}_{2}}{\bar{U}_{1}+a} \geq 0 \\
\geq-\epsilon_{1} \Delta \underline{U}_{1}-\underline{U}_{1}\left(1-\underline{U}_{1}\right)-\frac{\underline{U}_{1} \underline{U}_{2}}{\underline{U}_{1}+a}, \\
-\epsilon_{2} \Delta \bar{U}_{2}-\frac{c \bar{U}_{1} \bar{U}_{2}}{\bar{U}_{1}+a}+b \bar{U}_{2}-\frac{\bar{U}_{2} \bar{U}_{3}}{\bar{U}_{2}+d} \geq 0 \\
\geq-\epsilon_{2} \Delta \underline{U}_{1}-\frac{c \underline{U}_{1} \underline{U}_{2}}{\underline{U}_{1}+a}+b \underline{U}_{2}+\frac{\underline{U}_{2} \underline{U}_{3}}{\underline{U}_{2}+d}, \\
-\epsilon_{3} \Delta \bar{U}_{3}-p \bar{U}_{3}-\frac{q \bar{U}_{3}^{2}}{\bar{U}_{2}+r} \geq 0 \\
\geq-\epsilon_{3} \Delta \underline{U}_{1}-p \underline{U}_{3}-\frac{q \underline{U}_{3}^{2}}{\underline{U}_{2}+r}, \\
\frac{\partial \bar{U}_{1}}{\partial \nu} \geq 0 \geq \frac{\partial \underline{U}_{1}}{\partial \nu}, \quad \text { on } \partial \Omega . \\
\frac{\partial \bar{U}_{2}}{\partial v} \geq 0 \geq \frac{\partial \underline{U}_{2}}{\partial v}, \\
\frac{\partial \bar{U}_{3}}{\partial v} \geq 0 \geq \frac{\partial \underline{U}_{3}}{\partial v}, \quad
\end{gathered}
$$

By fixing $\bar{U}_{1}=1>\underline{U}_{1}=m_{1}$ and $\underline{U}_{2}=m_{2}$, according to Theorem 3 and under hypothesis $\left(H_{1}\right)$, we have $\bar{U}_{2} \leq c+c / 4 b$, and $c+c / 4 b \leq\left(\left(m_{1}+a\right) / m_{1}\right)\left[m_{2} /(1+a)+m_{1}\left(1-m_{1}\right)\right]$. This implies that $\bar{U}_{2} \leq\left(\left(m_{1}+a\right) / m_{1}\right)\left[m_{2} /(1+a)+m_{1}\left(1-m_{1}\right)\right]$. Thus, the right-hand term of the first inequality of (20) is satisfied. Let $\bar{U}_{1}=1$, and to satisfy the left term of the second inequality of system (20), we need $\underline{U}_{3}$ to satisfy the following inequality: $c /(1+a)-b-\underline{U}_{3} /\left(\bar{U}_{2}+d\right) \leq 0$. Let $\underline{U}_{1}=m_{1}$ and $\underline{U}_{2}=m_{2}$. According to Theorem 3 and under hypothesis $\left(H_{2}\right)$, we have $0 \leq \bar{U}_{3} \leq 1 / \alpha+c / 4 b \alpha+M / \alpha$ and $1 / \alpha+c / 4 b \alpha+M / \alpha \leq$ $c m_{1} /\left(m_{1}+a\right)-b-\bar{U}_{3} /\left(m_{2}+d\right)$.

Therefore,

$$
\frac{c m_{1}}{m_{1}+a}-b-\frac{\bar{U}_{3}}{m_{2}+d} \geq 0 .
$$

Thus, the right-hand side of the second inequality of system (20) is satisfied. To satisfy the right term of the third inequality of system (20), we need $\underline{U}_{3} \leq(p / q)\left(m_{2}+r\right)$. From Theorem 3 and hypothesis $\left(H_{3}\right)$, this last condition is satisfied.

3.4. Stability of Homogeneous Stationary Solutions. Let us consider a solution $U(x, t)$ of system $(7)$ in the following form:

$$
\begin{aligned}
U(x, t)= & \left(U_{1}(x, t), U_{2}(x, t), U_{3}(x, t)\right) \\
= & U_{s}(x)+J(x, t) \\
= & \left(U_{1 s}(x), U_{2 s}(x), U_{2 s}(x)\right) \\
& +\left(J_{1}(x, t), J_{2}(x, t), J_{3}(x, t)\right),
\end{aligned}
$$


where $U_{s}(x)$ is the positive solution of (17). Let us replace $U(x, t)$ by $(22)$ in system (10) and, by identifying all terms that are linearized in $J$, we have

$$
\frac{\partial J}{\partial t}=\left(\begin{array}{ccc}
\epsilon_{1} & 0 & 0 \\
0 & \epsilon_{2} & 0 \\
0 & 0 & \epsilon_{3}
\end{array}\right) \Delta J+\left(\begin{array}{ccc}
F_{U_{1}}^{*} & F_{U_{2}}^{*} & F_{U_{3}}^{*} \\
G_{U_{1}}^{*} & G_{U_{2}}^{*} & G_{U_{3}}^{*} \\
H_{U_{1}}^{*} & H_{U_{2}}^{*} & H_{U_{3}}^{*}
\end{array}\right) J,
$$

with

$$
\begin{aligned}
& F_{U_{1}}^{*}=1-2 U_{1}-\frac{a U_{2}}{\left(U_{1}+a\right)^{2}}, \\
& F_{U_{2}}^{*}=-\frac{U_{1}}{U_{1}+a}, \\
& F_{U_{3}}^{*}=0, \\
& G_{U_{1}}^{*}=\frac{a c U_{2}}{\left(U_{1}+a\right)^{2}}, \\
& G_{U_{2}}^{*}=\frac{c U_{1}}{U_{1}+a}-b-\frac{d U_{3}}{\left(U_{2}+a\right)^{2}}, \\
& G_{U_{3}}^{*}=-\frac{U_{2}}{U_{2}+d}, \\
& H_{U_{1}}^{*}=0, \\
& H_{U_{2}}^{*}=\frac{q U_{3}^{2}}{\left(U_{2}+d\right)^{2}}, \\
& H_{U_{3}}^{*}=p-\frac{2 q U_{3}}{U_{2}+d} .
\end{aligned}
$$

We assume the following assumptions:

$$
\begin{array}{cl}
\left(H_{4}\right) \quad F_{U_{1}}^{*}+G_{U_{2}}+H_{U_{3}}^{*}<0, & \\
\left(H_{5}\right) \quad & F_{U_{1}}^{*} G_{U_{2}}^{*}+F_{U_{1}}^{*} H_{U_{3}}^{*}+G_{U_{2}}^{*} H_{U_{3}}^{*}-F_{U_{2}}^{*} G_{U_{1}}^{*} \\
& \quad-F_{U_{3}}^{*} H_{U_{1}}^{*}-G_{U_{3}}^{*} H_{U_{2}}^{*}>0, \\
\left(H_{6}\right) \quad F_{U_{1}}^{*} G_{U_{3}}^{*} H_{U_{2}}^{*}+F_{U_{2}}^{*} G_{U_{1}}^{*} H_{U_{3}}^{*}+F_{U_{3}}^{*} G_{U_{2}}^{*} H_{U_{1}}^{*} \\
\quad-F_{U_{1}}^{*} G_{U_{2}}^{*} H_{U_{3}}^{*}-F_{U_{2}}^{*} G_{U_{3}}^{*} H_{U_{1}}^{*}-F_{U_{3}}^{*} G_{U_{1}}^{*} H_{U_{2}}^{*} \\
>0 .
\end{array}
$$

Theorem 6. Let $\left(H_{4}\right),\left(H_{5}\right)$, and $\left(H_{6}\right)$ hold; then the equilibrium $U_{s}$ is locally stable.

Proof. Concerning the proof of this theorem, we use the results established in $[22,23]$. In fact, we consider the following system:

$$
\begin{aligned}
-\Delta \chi_{j} & =\beta_{j} \chi_{j} \quad \text { on } \Omega, \\
\left.\frac{\partial \chi_{j}}{\partial \nu}\right|_{\partial \Omega} & =0,
\end{aligned}
$$

where $\chi_{j}$ is the eigenfunction of $-\Delta$ in $\Omega$ and the eigenvalue $\beta_{j}$ satisfying $0<\beta_{0}<\cdots<\beta_{j}<\cdots$.

So, we have

$$
J=\sum_{j=0}^{\infty} J_{j}(t) \chi_{j}(x, y), \quad J_{j}(t) \in \mathbb{R}^{n} .
$$

From (27) and (23), we have $\dot{J}_{j}=C_{j} J_{j}$, where $C_{j}$ is the matrix defined as

$$
C_{j}=\left(\begin{array}{ccc}
F_{U_{1}}^{*} & F_{U_{2}}^{*} & F_{U_{3}}^{*} \\
G_{U_{1}}^{*} & G_{U_{2}}^{*} & G_{U_{3}}^{*} \\
H_{U_{1}}^{*} & H_{U_{2}}^{*} & H_{U_{3}}^{*}
\end{array}\right)-\lambda_{j}\left(\begin{array}{ccc}
\epsilon_{1} & 0 & 0 \\
0 & \epsilon_{2} & 0 \\
0 & 0 & \epsilon_{3}
\end{array}\right) .
$$

$U_{s}$ is stable if and only if each $J_{j}(t)$ decreases to zero. So, $C_{j}$ has three eigenvalues with negative real parts $\xi_{k}, k=1,2,3$, such that $\xi^{3}+a \xi^{2}+b \xi+c=0$, with

$$
\begin{aligned}
a= & \beta_{j}\left(\epsilon_{1}+\epsilon_{2}+\epsilon_{3}\right)-\left(F_{U_{1}}^{*}+G_{U_{2}}^{*}+H_{U_{3}}^{*}\right), \\
b= & \beta_{j}^{2}\left(\epsilon_{1} \epsilon_{2}+\epsilon_{1} \epsilon_{3}+\epsilon_{2} \epsilon_{3}\right)-\beta_{j}\left(\epsilon_{1} G_{U_{2}}^{*}+\epsilon_{1} H_{U_{3}}^{*}\right. \\
& \left.+\epsilon_{2} F_{U_{1}}^{*}+\epsilon_{2} H_{U_{3}}^{*}+\epsilon_{3} F_{U_{1}}^{*}+\epsilon_{3} G_{U_{2}}^{*}\right)+F_{U_{1}}^{*} G_{U_{2}}^{*} \\
& +F_{U_{1}}^{*} H_{U_{3}}^{*}+G_{U_{2}}^{*} H_{U_{3}}^{*}-F_{U_{2}}^{*} G_{U_{1}}^{*}-F_{U_{3}}^{*} H_{U_{1}}^{*} \\
& +G_{U_{3}}^{*} H_{U_{2}}^{*}, \\
c= & \lambda_{j}^{3} \epsilon_{1} \epsilon_{2} \epsilon_{3}-\beta_{j}^{2}\left(\epsilon_{1} \epsilon_{2} H_{U_{3}}^{*}+\epsilon_{1} \epsilon_{3} G_{U_{2}}^{*}+\epsilon_{2} \epsilon_{3} F_{U_{1}}^{*}\right) \\
& +\beta_{j}\left(\epsilon_{1} G_{U_{2}} H_{U_{3}}^{*}+\epsilon_{2} F_{U_{1}}^{*} H_{U_{3}}^{*}+\epsilon_{3} F_{U_{1}}^{*} G_{U_{2}}^{*}\right. \\
& \left.-\epsilon_{1} G_{U_{3}}^{*} H_{U_{2}}^{*}-\epsilon_{2} F_{U_{3}}^{*} H_{U_{1}}^{*}-\epsilon_{3} F_{U_{2}}^{*} G_{U_{1}}^{*}\right) \\
& +F_{U_{1}}^{*} G_{U_{3}}^{*} H_{U_{2}}^{*}+F_{U_{2}}^{*} G_{U_{1}}^{*} H_{U_{3}}^{*}+F_{U_{3}}^{*} G_{U_{2}}^{*} H_{U_{1}}^{*} \\
& -F_{U_{1}}^{*} G_{U_{2}}^{*} H_{U_{3}}^{*}-F_{U_{2}}^{*} G_{U_{3}}^{*} H_{U_{1}}^{*}-F_{U_{3}}^{*} G_{U_{1}}^{*} H_{U_{2}}^{*} .
\end{aligned}
$$

For the three eigenvalues to admit a negative real part, we must necessarily have

$$
\begin{aligned}
& F_{U_{1}}^{*}+G_{U_{2}}^{*}+H_{U_{3}}^{*}-\beta_{j}\left(\epsilon_{1}+\epsilon_{2}+\epsilon_{3}\right)<0, \\
& F_{U_{1}}^{*} G_{U_{2}}^{*}+F_{U_{1}}^{*} H_{U_{3}}^{*}+G_{U_{2}}^{*} H_{U_{3}}^{*}-F_{U_{2}}^{*} G_{U_{1}}^{*}-F_{U_{3}}^{*} H_{U_{1}}^{*} \\
& \quad+G_{U_{3}}^{*} H_{U_{2}}^{*}-\beta_{j}\left(\epsilon_{1} G_{U_{2}}^{*}+\epsilon_{1} H_{U_{3}}^{*}+\epsilon_{2} F_{U_{1}}^{*}+\epsilon_{2} H_{U_{3}}^{*}\right. \\
& \left.\quad+\epsilon_{3} F_{U_{1}}^{*}+\epsilon_{3} G_{U_{2}}^{*}\right)+\beta_{j}^{2}\left(\epsilon_{1} \epsilon_{2}+\epsilon_{1} \epsilon_{3}+\epsilon_{2} \epsilon_{3}\right)>0, \\
& F_{U_{1}}^{*} G_{U_{3}}^{*} H_{U_{2}}^{*}+F_{U_{2}}^{*} G_{U_{1}}^{*} H_{U_{3}}^{*}+F_{U_{3}}^{*} G_{U_{2}}^{*} H_{U_{1}}^{*}-F_{U_{1}}^{*} G_{U_{2}}^{*} H_{U_{3}}^{*} \\
& \quad-F_{U_{2}}^{*} G_{U_{3}}^{*} H_{U_{1}}^{*}-F_{U_{3}}^{*} G_{U_{1}}^{*} H_{U_{2}}^{*}+\beta_{j}\left(\epsilon_{1} G_{U_{2}}^{*} H_{U_{3}}^{*}\right. \\
& \quad+\epsilon_{2} F_{U_{1}}^{*} H_{U_{3}}^{*}+\epsilon_{3} F_{U_{1}}^{*} G_{U_{2}}^{*}-\epsilon_{1} G_{U_{3}}^{*} H_{U_{2}}^{*}-\epsilon_{2} F_{U_{3}}^{*} H_{U_{1}}^{*} \\
& \left.\quad-\epsilon_{3} F_{U_{2}}^{*} G_{U_{1}}^{*}\right)-\beta_{j}^{2}\left(\epsilon_{1} \epsilon_{2} H_{U_{3}}^{*}+\epsilon_{1} \epsilon_{3} G_{U_{2}}^{*}+\epsilon_{2} \epsilon_{3} F_{U_{1}}^{*}\right) \\
& \quad+\beta_{j}^{3} \epsilon_{1} \epsilon_{2} \epsilon_{3}>0 .
\end{aligned}
$$


For $j=0$, from (30), we have

$$
\begin{aligned}
& F_{U_{1}}^{*}+G_{U_{2}}^{*}+H_{U_{3}}^{*}<0, \\
& F_{U_{1}}^{*} G_{U_{2}}^{*}+F_{U_{1}}^{*} H_{U_{3}}^{*}+G_{U_{2}}^{*} H_{U_{3}}^{*}-F_{U_{2}}^{*} G_{U_{1}}^{*}-F_{U_{3}}^{*} H_{U_{1}}^{*} \\
& \quad+G_{U_{3}}^{*} H_{U_{2}}^{*}>0, \\
& F_{U_{1}}^{*} G_{U_{3}}^{*} H_{U_{2}}^{*}+F_{U_{2}}^{*} G_{U_{1}}^{*} H_{U_{3}}^{*}+F_{U_{3}}^{*} G_{U_{2}}^{*} H_{U_{1}}^{*}-F_{U_{1}}^{*} G_{U_{2}}^{*} H_{U_{3}}^{*} \\
& \quad-F_{U_{2}}^{*} G_{U_{3}}^{*} H_{U_{1}}^{*}-F_{U_{3}}^{*} G_{U_{1}}^{*} H_{U_{2}}^{*}>0 .
\end{aligned}
$$

According to the Routh-Hurwitz criterion [24], sufficient stability conditions for positive stationary solutions can be obtained.

We will establish the stability of the homogeneous equilibrium $\left(U_{1}^{*}, U_{2}^{*}, U_{3}^{*}\right)$ under certain conditions. In fact, we have the following hypothesis:

$$
\begin{aligned}
\left(H_{7}\right) \quad & b<c, \\
& 1-a<U_{1}^{*}<\frac{a b}{c-b}, \\
& U_{2}^{*}<d(c-b) .
\end{aligned}
$$

Thus, we have the following theorem.

Theorem 7. Suppose that the hypotheses $\left(H_{a}\right),\left(H_{1}\right),\left(H_{2}\right)$, and $\left(H_{7}\right)$ are satisfied; then $\left(U_{1}^{*}, U_{2}^{*}, U_{3}^{*}\right)$ is globally asymptotically stable.

Proof. Let us define the function

$$
\begin{gathered}
\Upsilon\left(U_{1}, U_{2}, U_{3}\right)=\int_{\Omega}\left(\int_{U_{1}^{*}}^{U_{1}} \frac{n-U_{1}^{*}}{\eta} d \eta\right. \\
\quad+\int_{U_{2}^{*}}^{U_{2}} \frac{\left(n-U_{2}^{*}\right)(\eta+d)}{\eta} d \eta \\
\left.+\int_{U_{3}^{*}}^{U_{3}} \frac{n-U_{3}^{*}}{\eta} d \eta\right) d x .
\end{gathered}
$$

We have

$$
\begin{aligned}
\frac{d \Upsilon}{d t} & =T_{1}+T_{2}, \\
T_{1} & =\int_{\Omega}\left[\frac{U_{1}-U_{1}^{*}}{U_{1}}\left(U_{1}\left(1-U_{1}\right)-\frac{U_{1} U_{2}}{U_{1}+a}\right)\right. \\
& +\frac{\left(U_{2}-U_{2}^{*}\right)\left(U_{2}+d\right)}{U_{2}}\left(\frac{c U_{1} U_{2}}{U_{1}+a}-b U_{2}-\frac{U_{2} U_{3}}{U_{2}+d}\right) \\
& \left.+\frac{U_{3}-U_{3}^{*}}{U_{3}}\left(p U_{3}-\frac{q U_{3}^{2}}{U_{2}+r}\right)\right] d x \\
& =\int_{\Omega}\left[\frac { U _ { 1 } - U _ { 1 } ^ { * } } { U _ { 1 } + a } \left(\left(1-U_{1}\right)\left(U_{1}+a\right)-U_{2}+U_{2}^{*}\right.\right. \\
& \left.-\left(1-U_{1}^{*}\right)\left(U_{1}^{*}+a\right)\right)
\end{aligned}
$$

$$
\begin{aligned}
& +\frac{\left(U_{2}-U_{2}^{*}\right)\left(U_{2}+d\right)}{U_{2}}\left(c U_{1}\left(U_{2}+d\right)\right. \\
& \left.-b\left(U_{1}+a\right)\left(U_{2}+d\right)-U_{3}\left(U_{1}+a\right)\right) \\
& -\left(c U_{1}^{*}\left(U_{2}^{*}+d\right)-b\left(U_{1}^{*}+a\right)\left(U_{2}^{*}+d\right)\right. \\
& \left.-U_{3}^{*}\left(U_{1}^{*}+a\right)\right)+\left(U_{2}-U_{2}^{*}\right)\left(\frac{q U_{3}^{*}}{U_{2}^{*}+r}\right. \\
& \left.\left.-\frac{q U_{3}}{U_{2}+r}\right)\right] d x=-\int_{\Omega}\left(U_{1}-U_{1}^{*}, U_{2}-U_{2}^{*}\right. \\
& \left.-U_{3}^{*}\right) S\left(U_{1}-U_{1}^{*}, U_{2}-U_{2}^{*}, U_{3}-U_{3}^{*}\right)^{T} d x,
\end{aligned}
$$

where

$S$

$$
=\left(\begin{array}{ccc}
\frac{U_{1}+U_{1}^{*}+a-1}{U_{1}+a} & \frac{U_{3}^{*}-(c-b)\left(U_{2}^{*}+d\right)}{U_{1}+a} & 0 \\
\frac{1}{U_{1}+a} & \frac{a b-(c-b) U_{1}^{*}}{U_{1}+a} & \frac{-p}{U_{2}+r} \\
0 & 1 & \frac{q}{U_{2}+r}
\end{array}\right) .
$$

Under hypothesis $\left(H_{7}\right)$, the matrix $S$ is positive, and consequently $T_{1}<0$.

On the other hand, we have

$$
\begin{aligned}
T_{2} & =\int_{\Omega}\left[\epsilon_{1} \frac{U_{1}-U_{1}^{*}}{U_{1}} \Delta U_{1}\right. \\
& +\epsilon_{2} \frac{\left(U_{2}-U_{2}^{*}\right)\left(U_{2}+d\right)}{U_{2}} \Delta U_{2} \\
& \left.+\epsilon_{3} \frac{\left(U_{3}-U_{3}^{*}\right)}{U_{3}} \Delta U_{3}\right] d x=-\epsilon_{1} \int_{\Omega}\left|\nabla U_{1}\right|^{2} \\
& \cdot \frac{U_{1}^{*}}{U_{1}} d x-\epsilon_{2} \int_{\Omega}\left|\nabla U_{2}\right|^{2} \frac{U_{2}+d U_{3}^{*}}{U_{2}} d x \\
& -\epsilon_{3} \int_{\Omega}\left|\nabla U_{3}\right|^{2} \frac{U_{3}^{*}}{U_{3}} d x<0 .
\end{aligned}
$$

\section{Numerical Experiments}

In this section, we perform extensive numerical simulations of the spatial model system (7) in two-dimensional space using the forward finite difference method. The set of parameter values used for the numerical simulation are given in Table $1[11,15-17,25]$. Here, the system is studied on a spatial domain: $\Omega=[0,50] \times[0,50]$. It is assumed that the fish, zooplankton, and phytoplankton are spread over the whole domain at the beginning. 

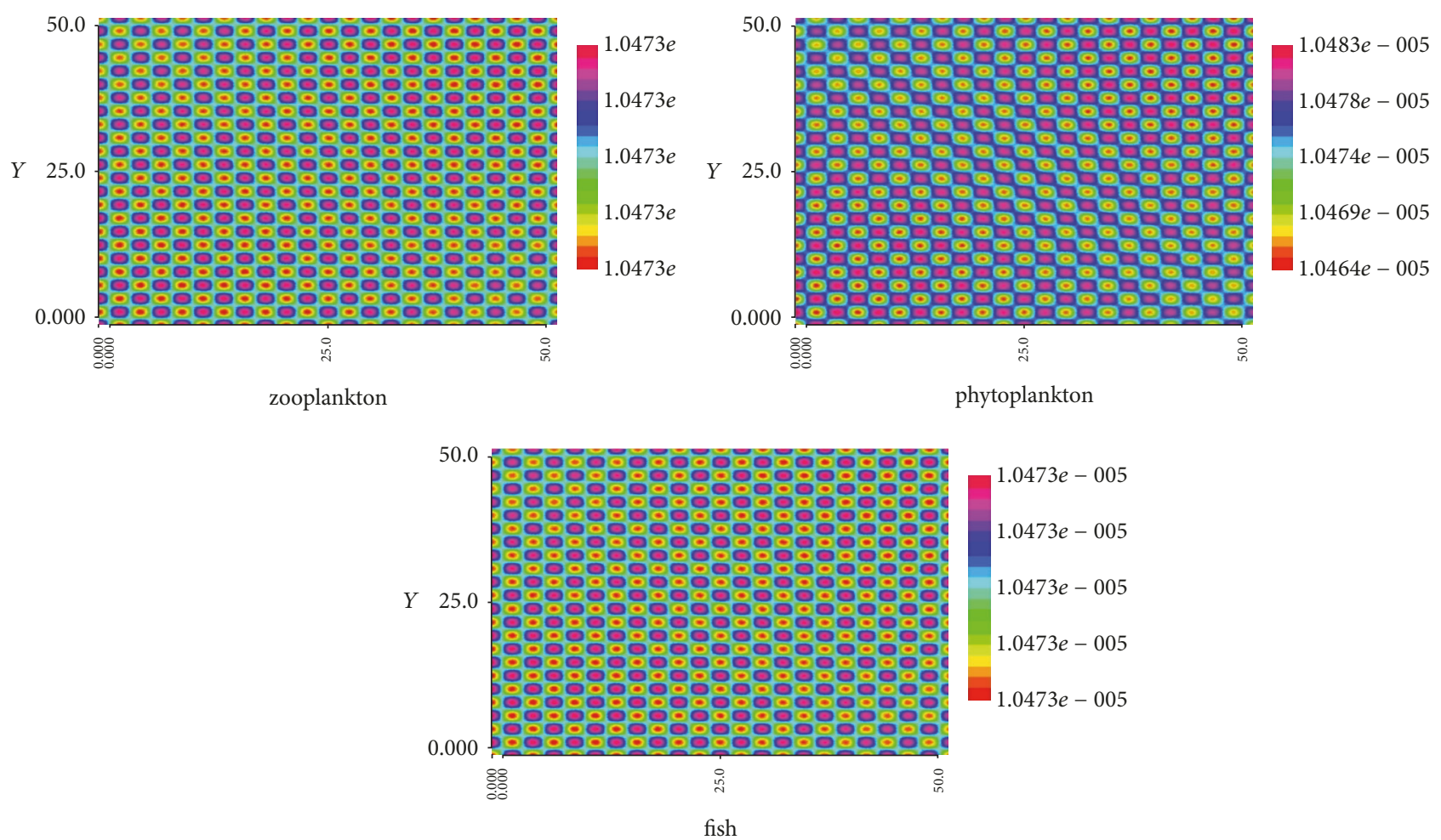

FIgURE 2: Spatial distribution of the system with $b_{0}=0.25$ and $\alpha_{0}=0.6$.
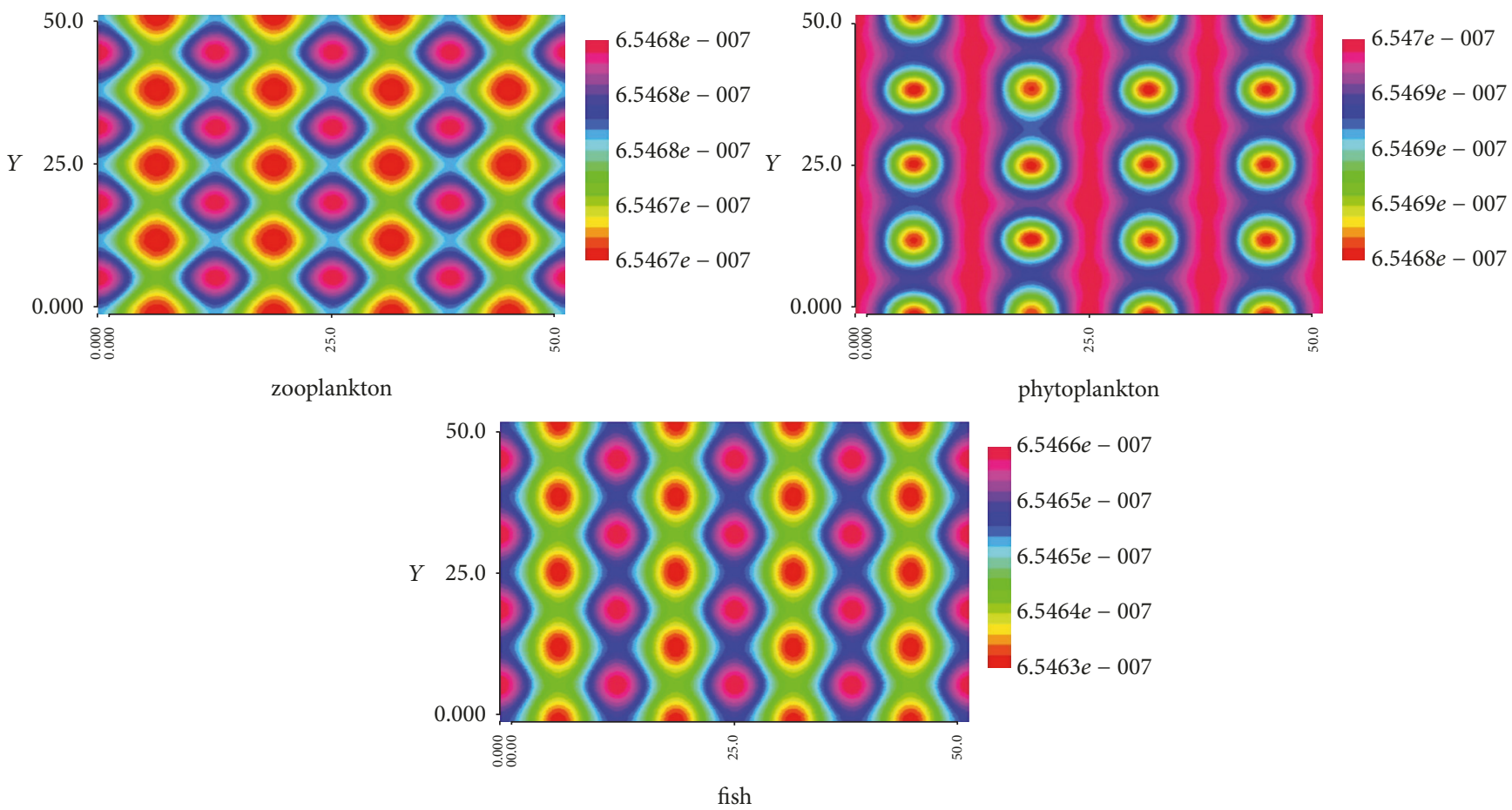

FIGURE 3: Spatial distribution of the system with $b_{0}=0.38$ and $\alpha_{0}=0.54$. 

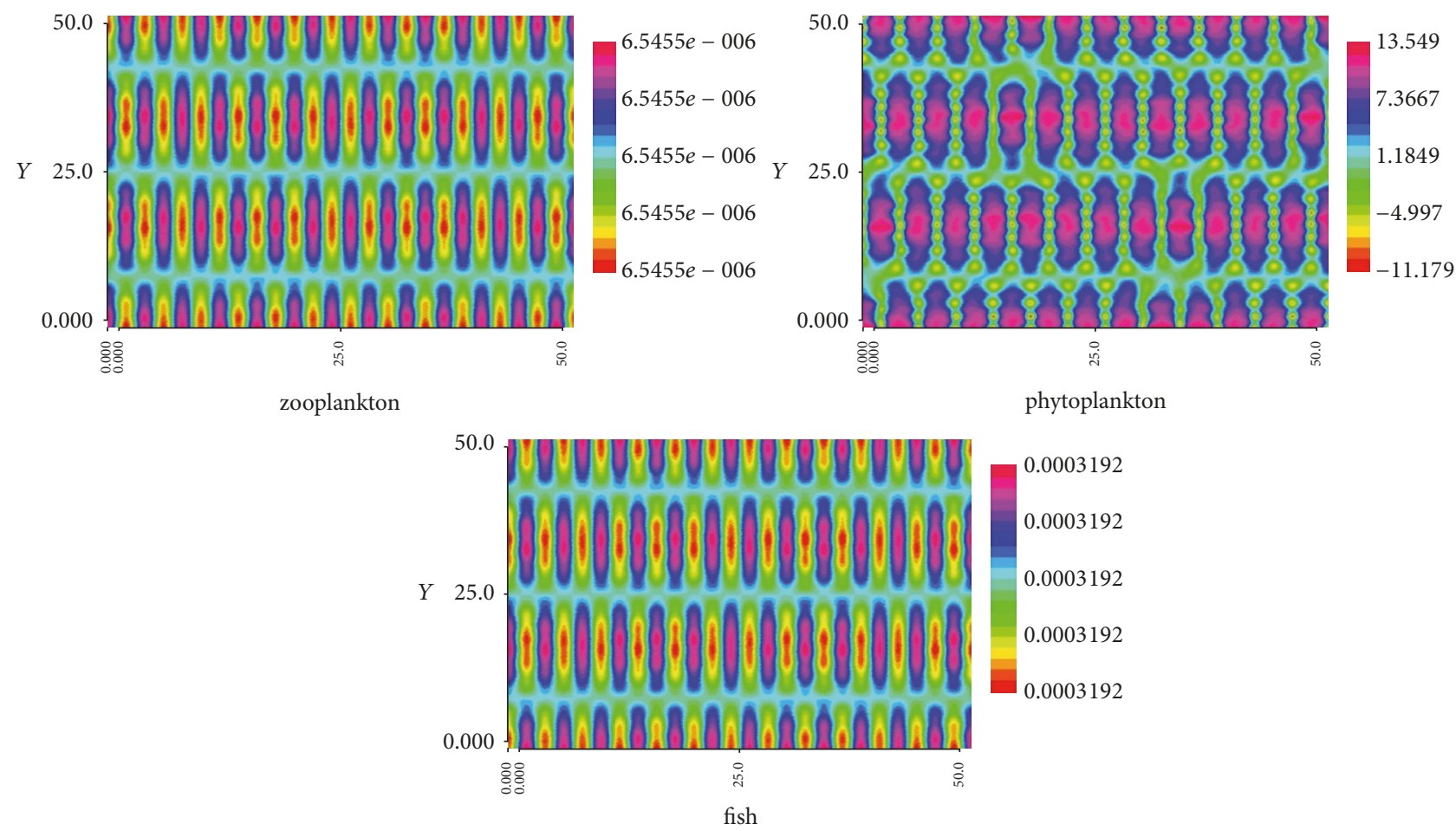

FIGURE 4: Spatial distribution of the system with $b_{0}=0.4032$ and $\alpha_{0}=0.342$.
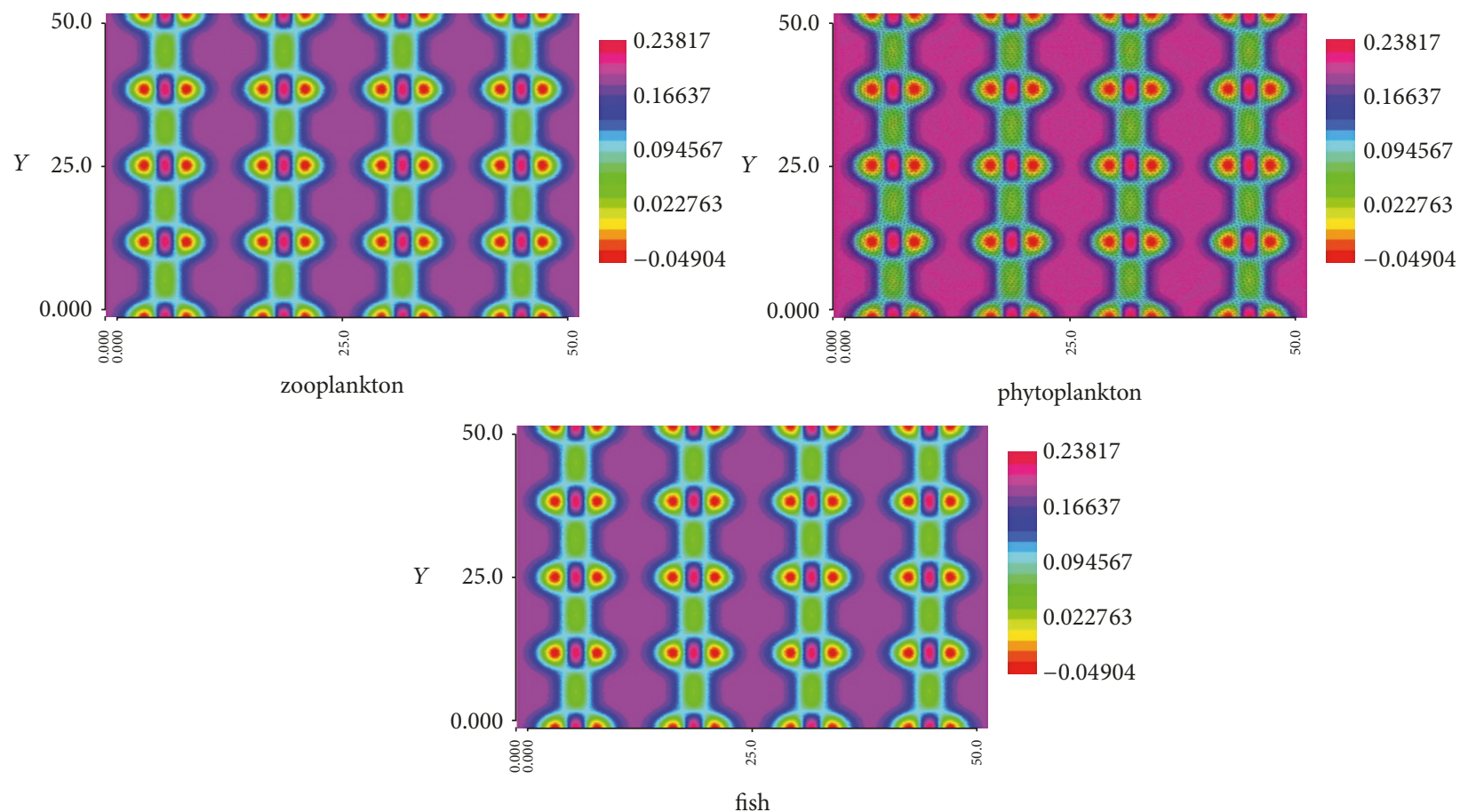

FIGURE 5: Spatial distribution of the system with $b_{0}=0.2562$ and $\alpha_{0}=0.71$. 

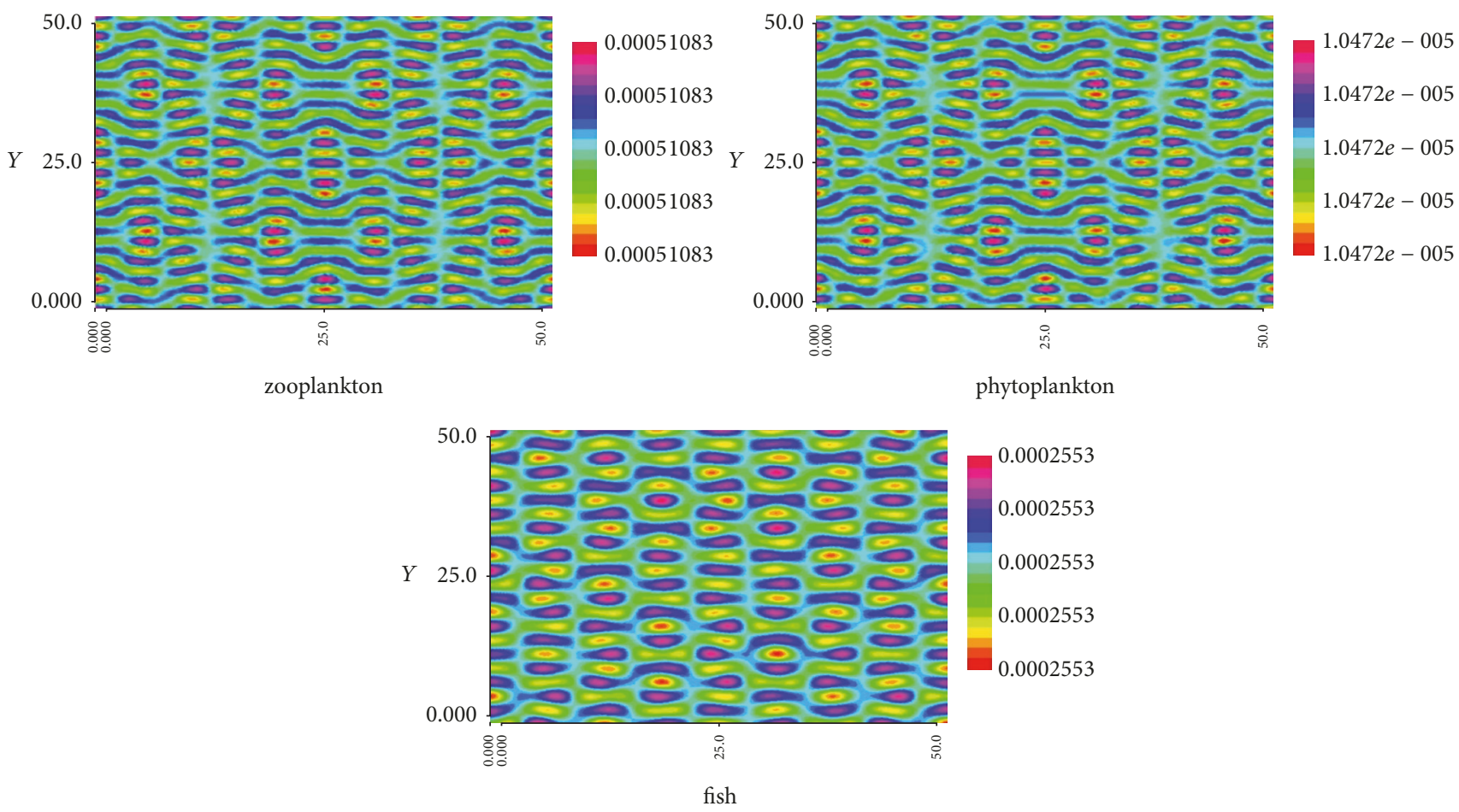

FIGURE 6: Spatial distribution of the system with $b_{0}=0.61$ and $\alpha_{0}=0.69$.
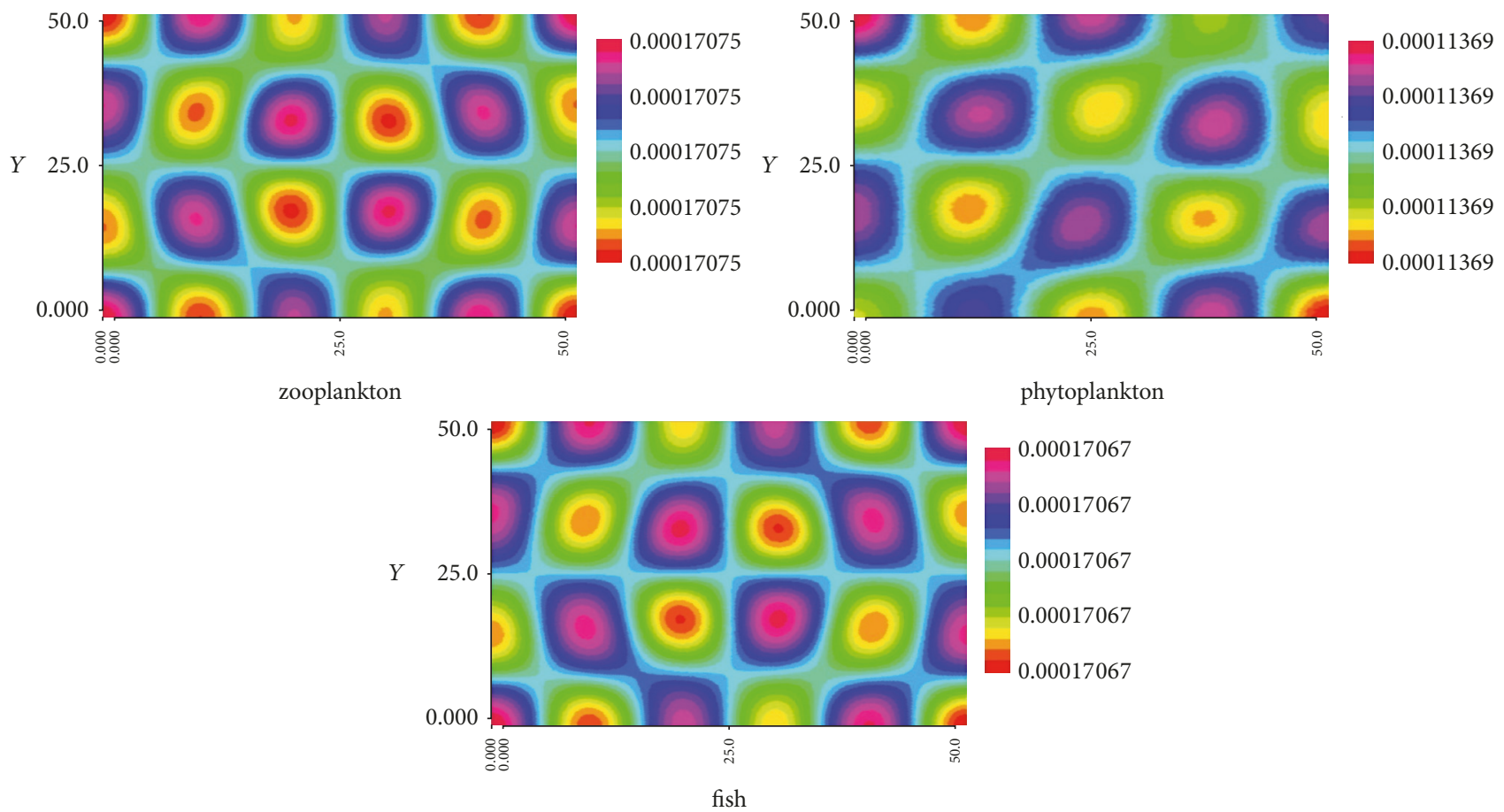

Figure 7: Spatial distribution of the system with $b_{0}=0.69$ and $\alpha_{0}=0.595$. 

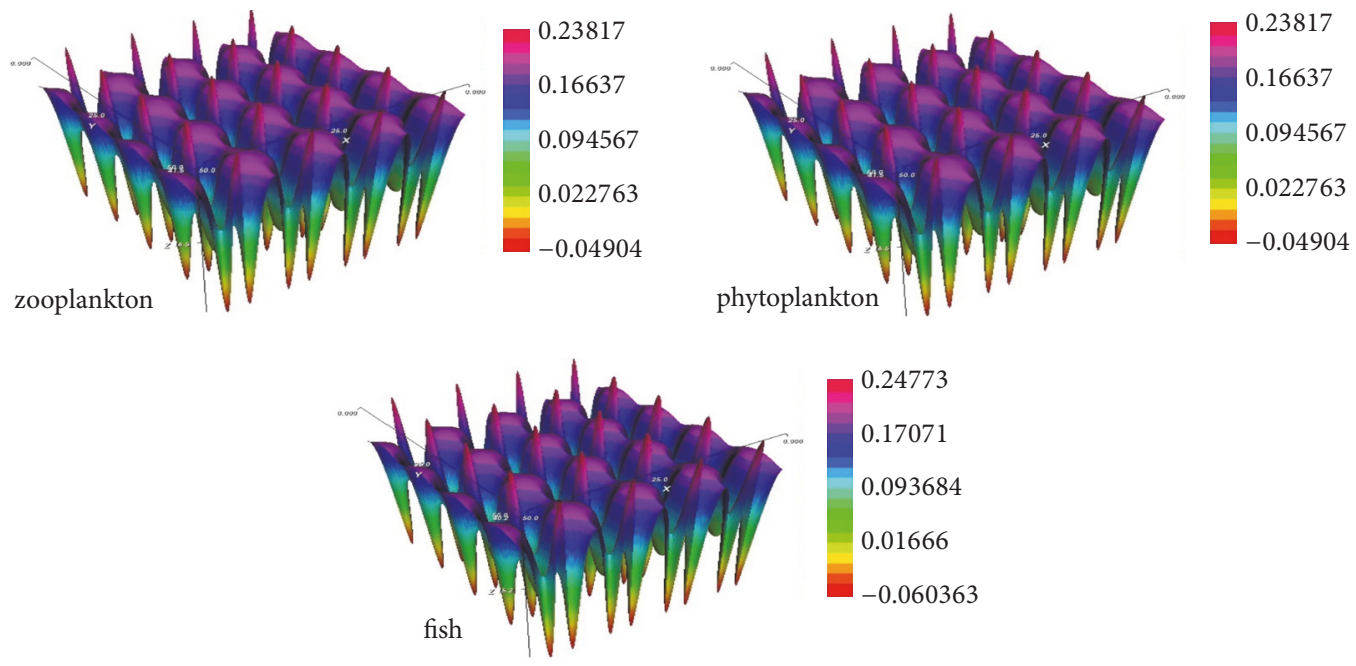

FIgURE 8: Dynamics behavior for $\theta_{p}=0.18$.

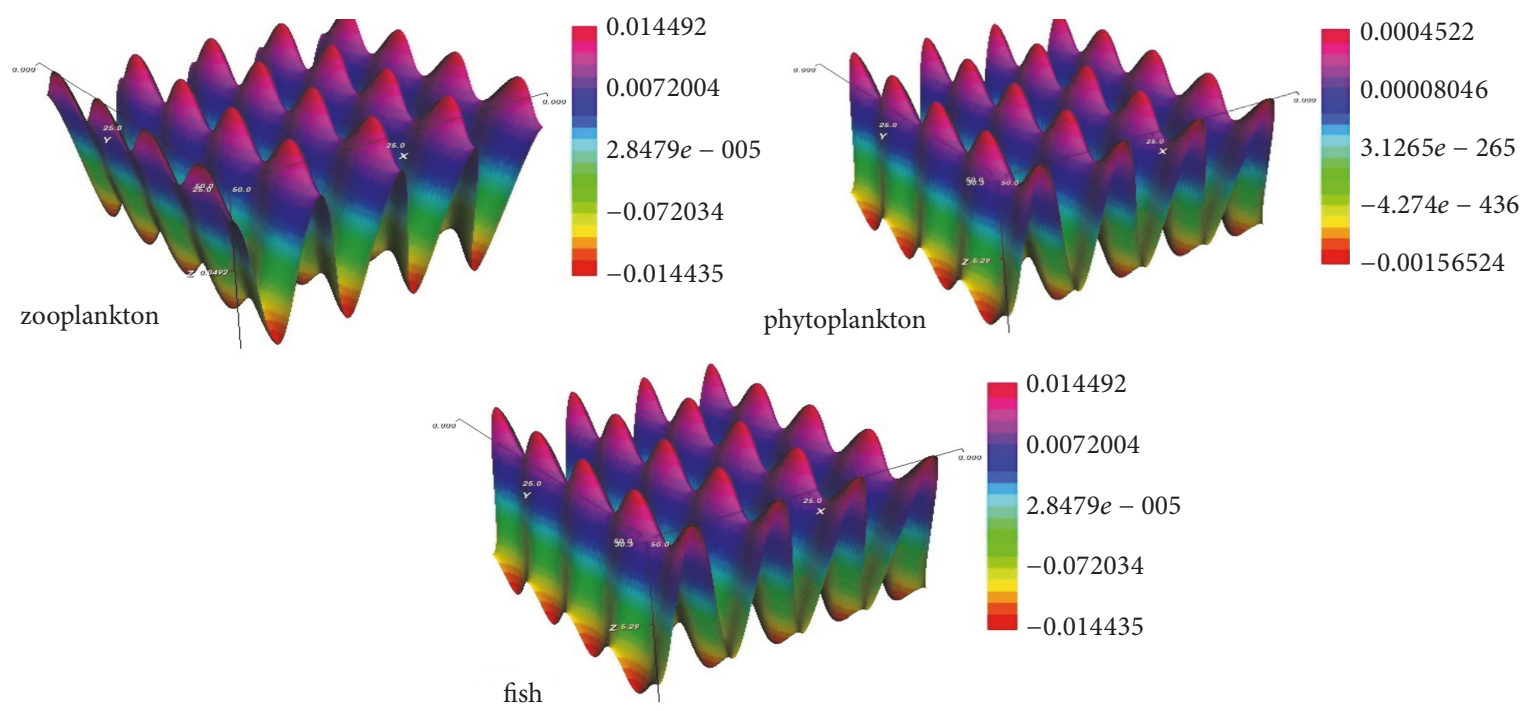

FIgURE 9: Dynamics behavior for $\theta_{p}=0.29$.
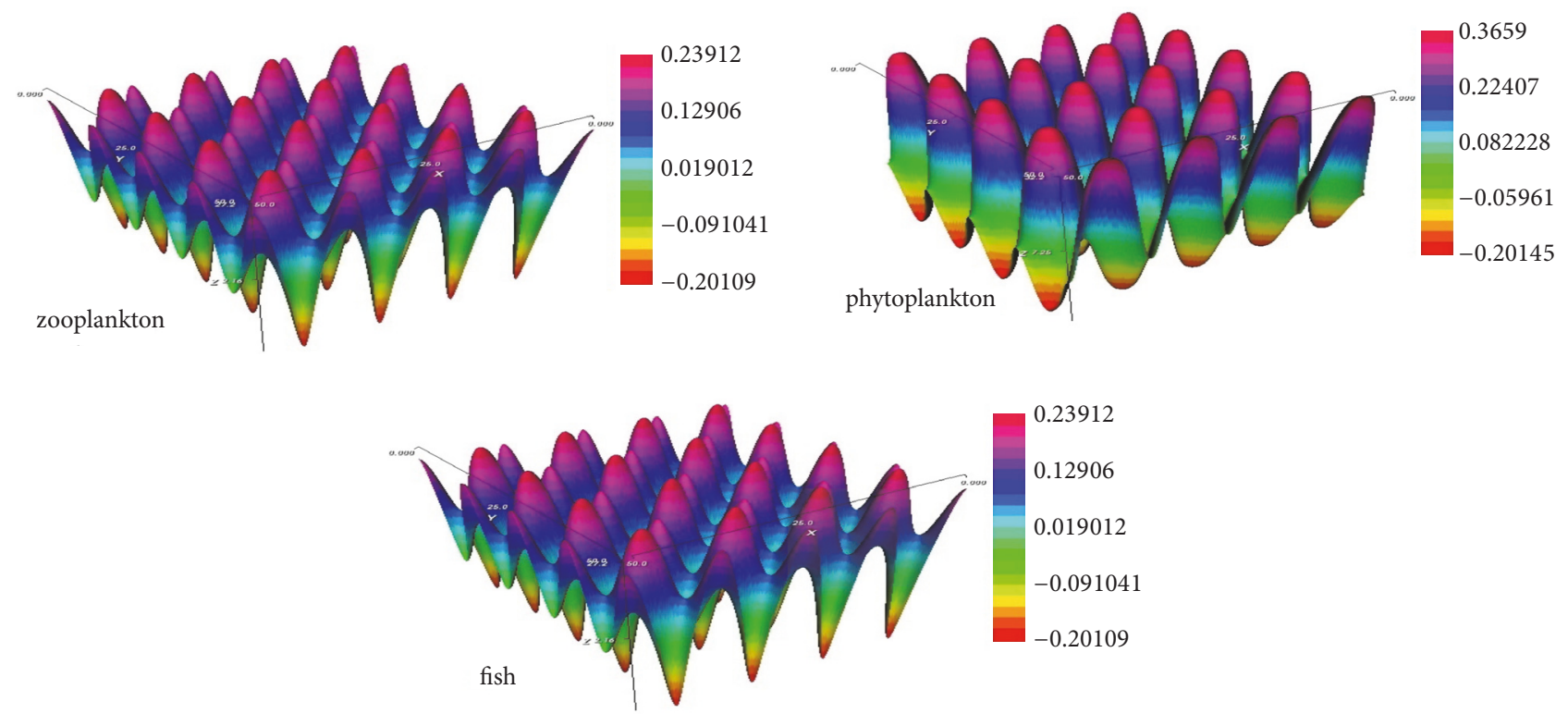

FIgURE 10: Dynamics behavior for $\theta_{p}=0.46$. 

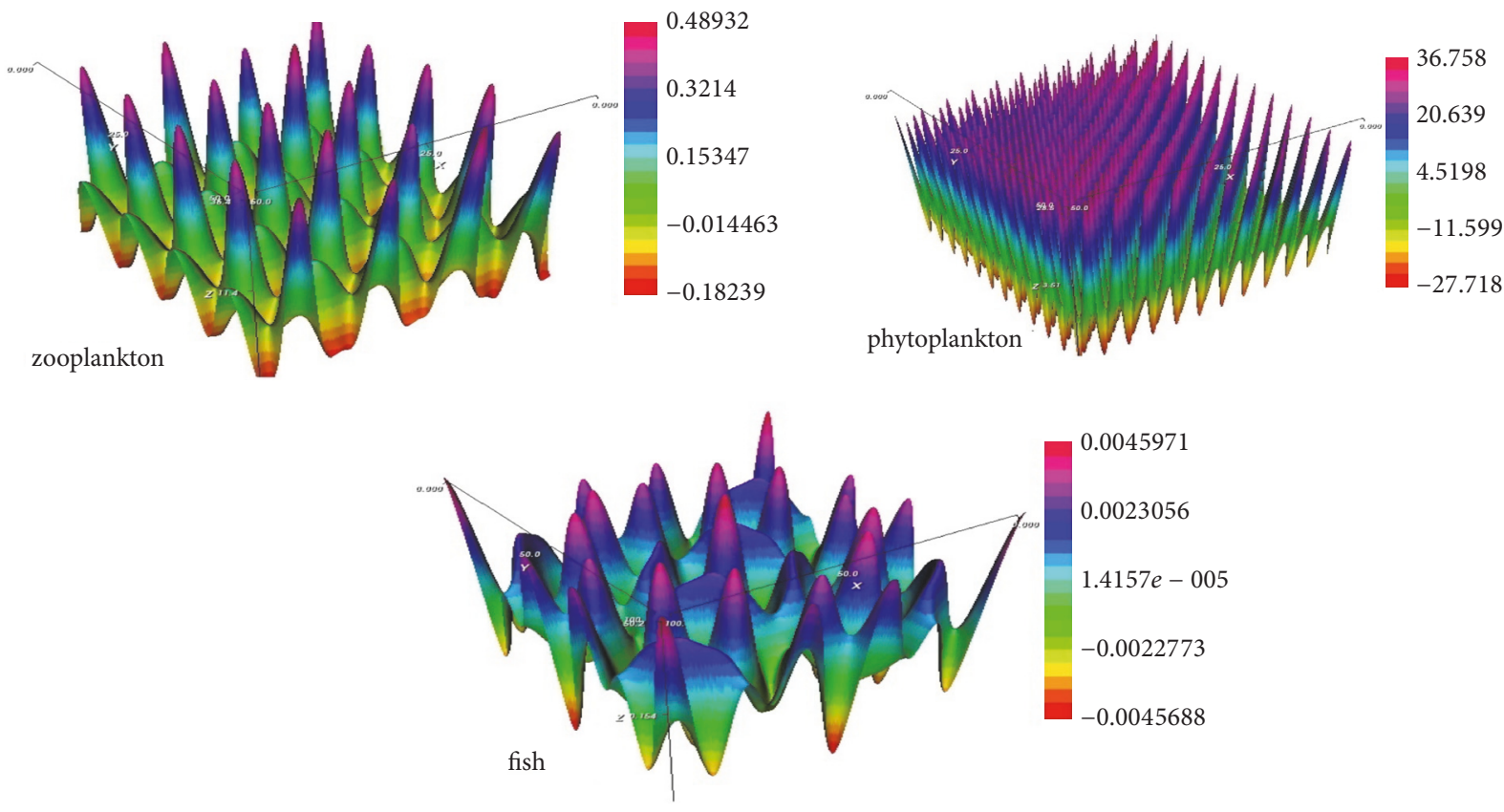

FIgURE 11: Dynamics behavior for $\theta_{p}=0.935$.

TABLE 1: Numerical values for the parameters of system.

\begin{tabular}{lcc}
\hline Parameter & Description & Value \\
\hline$\gamma_{0}$ & Half-saturation constant of the $Z$ predation on $P$ & 0.7 \\
$\gamma_{2}$ & The value of $Z$ (elimination rate by the individual of $Z$ ) & 0.4 \\
$\gamma_{3}$ & The residual loss caused by high rarity of $Z$ of individuals of $F$ & 0.9 \\
$r_{p}$ & Growth rate of the phytoplankton population & 1.6 \\
$r_{z}$ & External mortality rate of the zooplankton population & 0.4 \\
$\delta_{1}$ & Diffusivity coefficient of the phytoplankton population & 0.03 \\
$\delta_{2}$ & Diffusivity coefficient of the zooplankton population & 0.03 \\
$\mu_{\epsilon}$ & External mortality rate of phytoplankton population & 0.9 \\
$\delta_{3}$ & Diffusion coefficient of the fish population & 0.05 \\
$\alpha_{1}$ & Consumption rate of the phytoplankton population by $Z$ & 0.75 \\
$\alpha_{2}$ & Consumption rate of the zooplankton population by $F$ & 0.4 \\
$\alpha_{3}$ & Mortality rate of the fish population due to the competition & 0.122 \\
$\mu_{f}$ & External mortality rate of the fish population \\
$r_{f}$ & Growth rate of the fish population & 0.02 \\
\hline
\end{tabular}

4.1. Pattern Formation. Here, we will illustrate the mathematical predictions, by numerical simulations, concerning the behavior of the dynamics under hypotheses $\left(H_{1}\right)-\left(H_{7}\right)$. The qualitative results of different pattern formations due to the variation of $b_{0}$ and $\alpha_{0}$ are shown. Here, we consider the value of released toxin $\theta_{p}=0.13$. These numerical results show that, for every strictly positive initial condition, under assumptions $\left(H_{1}\right)-\left(H_{7}\right)$, the nonhomogeneous equilibrium is always globally asymptotically stable. Figures $2-7$ show the spatial structures formation for the threes species described in (7). These numerical results confirm the mathematical results about the existence and the stability of positive equilibrium according to the values of $b_{0}$ and $\alpha_{0}$. In this case, we will speak of a subsistence phenomenon of the fish and zooplankton population.

Remark 8. From a biological point of view, these results (Figures 2-7) show that there is coexistence between the three populations despite the release of the toxin into the aquatic environment. This means that, despite the harmful effects of the toxin released by phytoplankton, fish and zooplankton populations persist. 


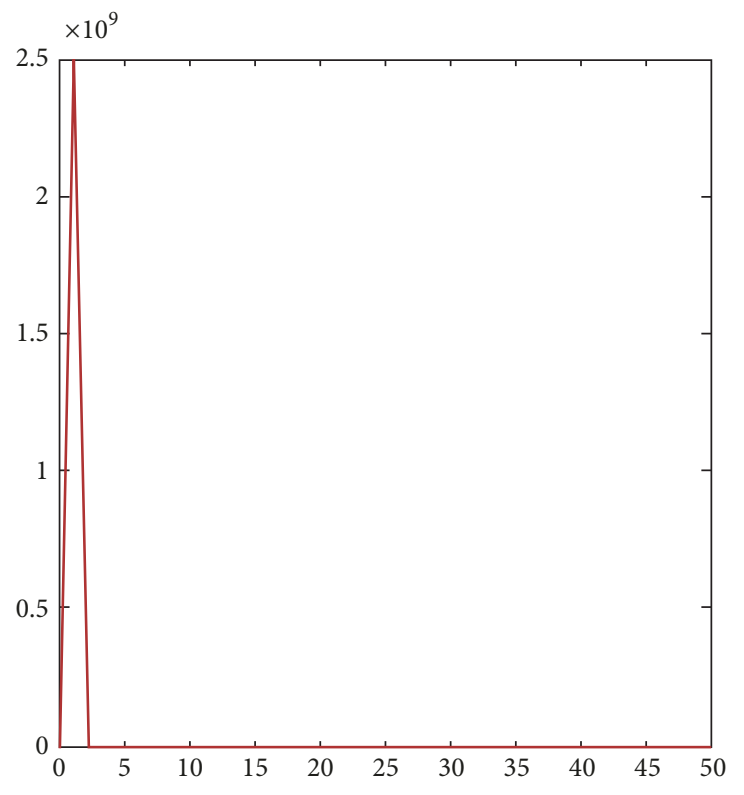

(a)

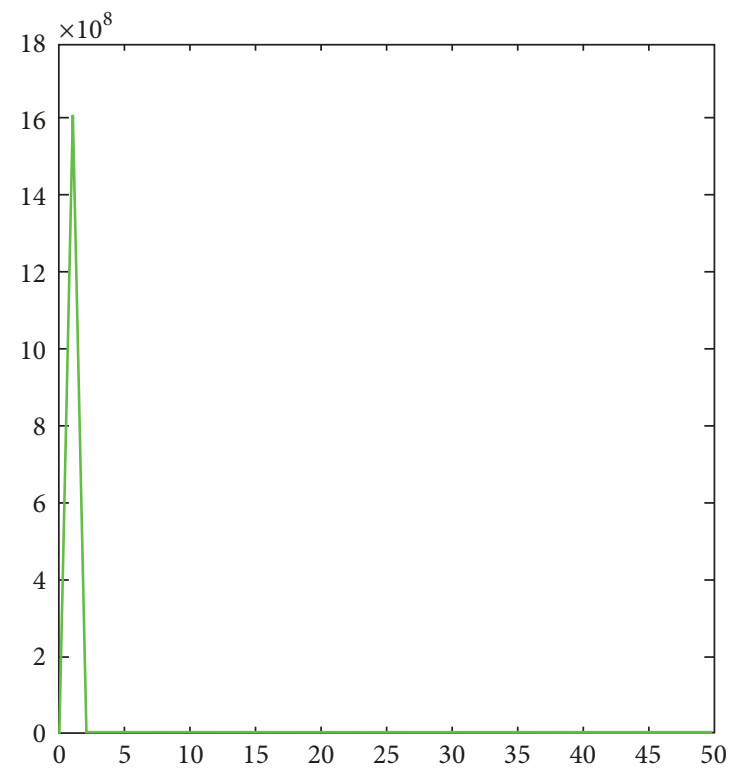

(b)

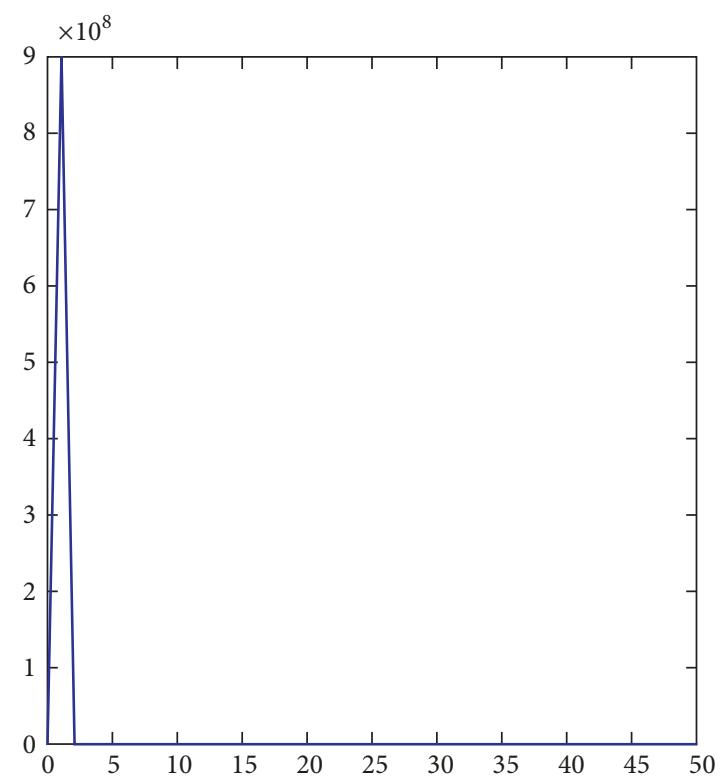

(c)

FIGURE 12: Corresponding convergence for error estimation in function of the iterations, where the simulated time is $t=50$ : (a) for $P$ (b) for $Z$, and (c) for $F$.

4.2. Analysis of the Dynamics Behavior with Toxin Effect. In this section, we consider that $b_{0}=0.2562$ and $\alpha_{0}=$ 0.71 . Figures $8-11$ show that, after a transitional phase, an equilibrium can be established with a strong distribution of the three populations, which is not far from the results obtained previously. As a biological interpretation, we can say that if the toxin is released below this value, the impact is not significant on the fish and zooplankton populations (Figures $8-10)$. A less dense distribution of fish and zooplankton than before is observed. This explains the considerable decrease of these species due to the increase in the number of toxic phytoplankton. On the other hand, we observe in Figure 11 a sudden change concerning the spatial distribution of phytoplankton (strong and bad spatial distribution).

Remark 9. Figure 12 shows the estimate of the error by using the standard norm $\|\cdot\|_{2}$ on the iterations $[16,17,26]$. These figures show a decrease in error estimation over the course of the iterations. This confirms the convergence of the numerical scheme used.

\section{Conclusion and Future Work}

Our interest in this paper is the construction of a reactiondiffusion model for modeling the dynamics of fish population 
under a diet on plankton (zooplankton and phytoplankton), taking into account the effect of the toxin. The model formulation is derived from an ODE system by considering an isotropic distribution as in [12]. It should be noted that we consider diffusion independently of the spatial variable in the construction of the reaction-diffusion model. The mathematical results allowed us to establish conditions of existence of equilibrium which depend on the demographic parameters. We have also given some results about the stability of the stationary equilibria and we have established the conditions on the inexistence of the equilibrium with strictly positive components. We continued our study through numerical experiments in order to confirm our mathematical results. Also, our numerical results have yielded interesting results on the effect of the toxin on the dynamics. This is why we are led to conclude that the release of the toxin under certain conditions in the aquatic space contributes to the regulation of the system. The phytoplankton bloom was observed during our simulations and is in perfect agreement with the biological observations.

Despite important results obtained in this paper, in order to further our study, we can consider for future work subdividing the phytoplankton population into toxic phytoplankton and nontoxic phytoplankton to extend our results to a system of cross-diffusion type.

\section{Conflicts of Interest}

The authors declare that there are no conflicts of interest.

\section{Authors' Contributions}

All the authors read and approved the final manuscript.

\section{References}

[1] M. Kot, Elements of Mathematical Biology, Cambridge University Press, Cambridge, UK, 2001.

[2] L. Matthews and J. Brindley, "Patchiness in plankton populations," Dynamics and Stability of Systems, vol. 12, no. 1, pp. 39-59, 1997.

[3] W. Ouedraogo, B. Sangaré, and S. Traoré, "A mathematical study of cannibalism in the fish-plankton model by taking into account the catching effect," Advanced Modeling and Optimization, vol. 18, no. 2, pp. 197-216, 2016.

[4] E. Briand, Contribution ã la comprehention du determinisme de la mise en place des proliferation du cyanobacteries et leur production de toxine [PhD. Thesis], Université Paris, 2008.

[5] M. L. Rosenzweig and R. H. MacArthur, "Graphical representation and stability conditions of predator-prey interactions," The American Naturalist, vol. 97, no. 895, pp. 209-223, 1963.

[6] J. Steele and E. Henderson, "The significance of interannual variability," in Towards a Model of Ocean Bio-geochemical Processes, G. T. Evans and M. J. R. Fasham, Eds., pp. 237-260, Springer, 1993.

[7] B. Traoré, B. Sangaré, and S. Traoré, "A mathematical model of Malaria transmission with structured vector population and seasonality," Journal of Applied Mathematics, vol. 2017, Article ID 6754097, 15 pages, 2017.
[8] A. M. Edward and J. Brindley, "Zooplankton mortality and the dynamical behavior of plankton population models," Bulletin of Mathematical Biology, pp. 303-339, 1999.

[9] C. Kohlmeier and W. Ebenhöh, "The stabilizing role of cannibalism in a predator-prey system," Bulletin of Mathematical Biology, vol. 57, no. 3, pp. 401-411, 1995.

[10] M. A. Aziz Alaoui and M. Daher Okaye, "Boundness and global stability for a predator-prey model with modified Leslie Gower and Holling-type 2," Applied Mathematical Letters, vol. 16, no. 7, pp. 1069-1075, 2003.

[11] W. Ouedraogo, B. Sangaré, and S. Traoré, "Some mathematical problems arising in biological models: a predator-prey model fish-plankton," Journal of Applied Mathematics \& Bioinformatics, vol. 5, no. 4, pp. 1-27, 2015.

[12] F. Courchamp, M. Langlais, and G. Sugihara, "Rabbits killing birds: modelling the hyperpredation process," Journal of Animal Ecology, vol. 69, no. 1, pp. 154-164, 2000.

[13] M. Bendahmane, "Analysis of a reaction-diffusion system modeling predator-prey with prey-taxis," Networks and Heterogeneous Media, vol. 3, no. 4, pp. 863-879, 2008.

[14] B. Ainseba, F. Heiser, and M. Langlais, "A mathematical analysis of a predator-prey system in a highly heterogeneous environment," Differential and Integral Equations, vol. 15, no. 4, pp. 385404, 2002.

[15] S. Gaucel, M. Langlais, and D. Pontier, "Invading introduced species in insular heterogeneous environments," Ecological Modelling, vol. 188, no. 1, pp. 62-75, 2005.

[16] B. Andreianov, M. Bendahmane, and R. Ruiz-Baier, "Analysis of a finite volume method for a cross-diffusion model in population dynamics," Mathematical Models and Methods in Applied Sciences, vol. 21, no. 2, pp. 307-344, 2011.

[17] G. Galiano, M. A. Garzon, and A. Jungel, "Semi-discretization in time and numerical convergence of solutions of a nonlinear cross-diffusion population model," Numerische Mathematik, vol. 93, no. 4, pp. 655-673, 2003.

[18] B. I. Camara, "Complexite de dynamiques de modeles proiepredateur avec difusion et applications. Mathematics," Mathematics Université du Havre, 2009 (French).

[19] M. Daher Okiye and M. A. Aziz-Alaoui, "On the dynamics of a predator-prey model with the Holling-Tanner functional response," in Proceedings of the ESMTB Conference, MIRIAM Editions, pp. 270-278, 2002.

[20] G. Kreisselmeier and R. Engel, "Nonlinear observers for autonomous Lipschitz continuous systems," Institute of Electrical and Electronics Engineers Transactions on Automatic Control, vol. 48, no. 3, pp. 451-464, 2003.

[21] H. Amann, "Dynamic theory of quasilinear parabolic equations. I. Abstract evolution equations," Nonlinear Analysis. Theory, Methods \& Applications, vol. 12, no. 9, pp. 895-919, 1988.

[22] D. G. Aronson and H. F. Weinberger, "Nonlinear diffusion in population genetics, combustion, and nerve pulse propagation," Lecture Notes in Math, vol. 446, pp. 5-49, 1975.

[23] J. Smoller, Shock Waves and Reaction-Diffusion Equations, Springer, New York, NY, USA, 1983.

[24] M. M. Fahmy and J. O’Reilly, "A note on the Routh-Hurwitz test," Institute of Electrical and Electronics Engineers Transactions on Automatic Control, vol. 27, no. 2, pp. 483-485, 1982.

[25] J. Dockery, V. Hutson, K. Mischaikow, and M. Pernarowski, "The evolution of slow dispersal rates: a reaction diffusion model," Journal of Mathematical Biology, vol. 37, no. 1, pp. 6183, 1998. 
[26] J. Pousin and J. Rappaz, "Consistency, stability, a priori and a posteriori errors for Petrov-Galerkin methods applied to nonlinear problems," Numerische Mathematik, vol. 69, no. 2, pp. 213-231, 1994. 


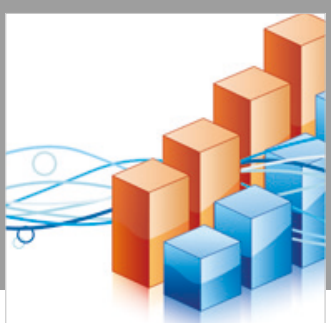

Advances in

Operations Research

\section{-n-m}
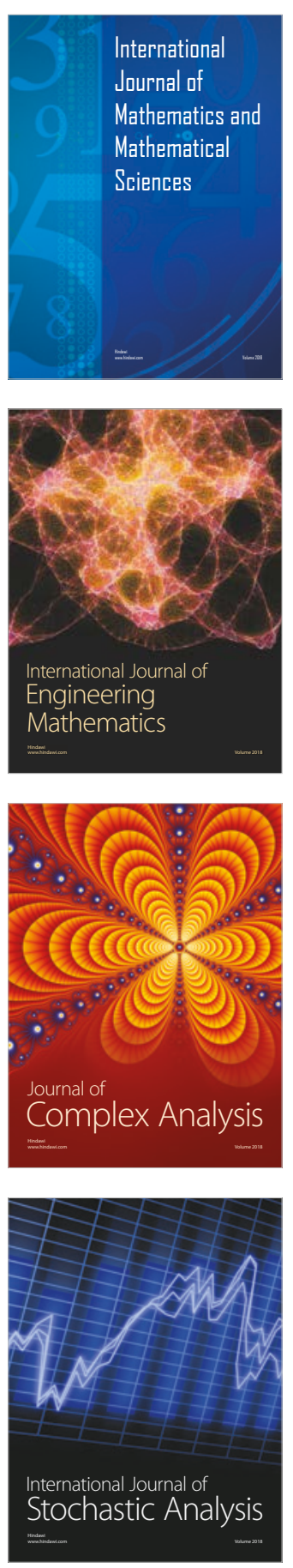
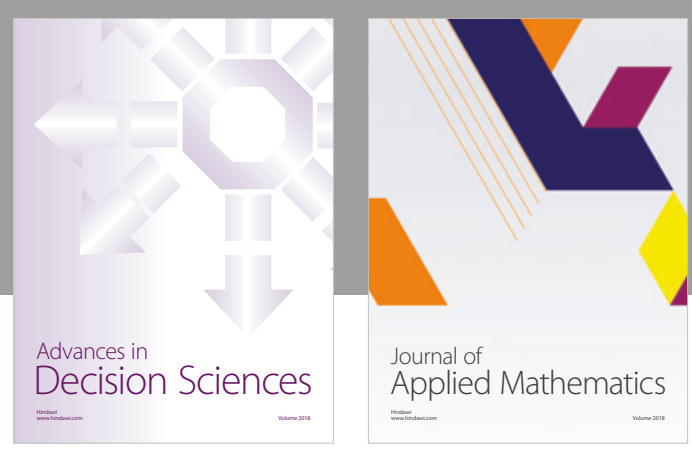

Journal of

Applied Mathematics
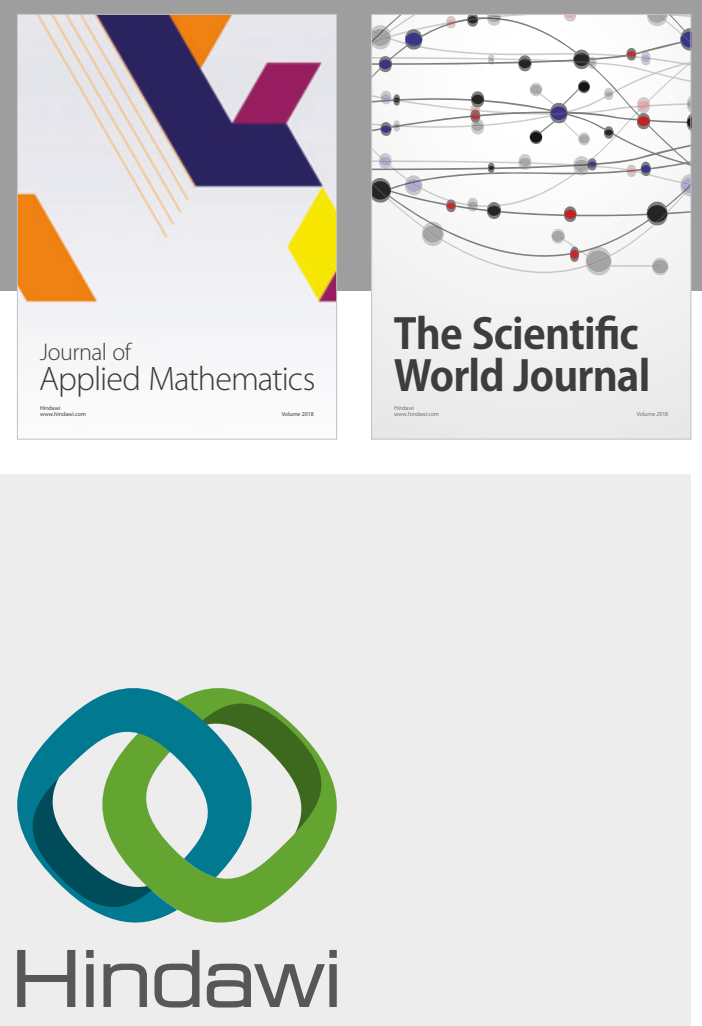

Submit your manuscripts at

www.hindawi.com

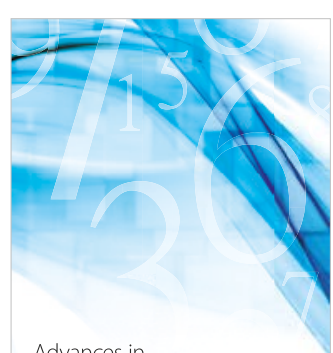

Advances in
Numerical Analysis
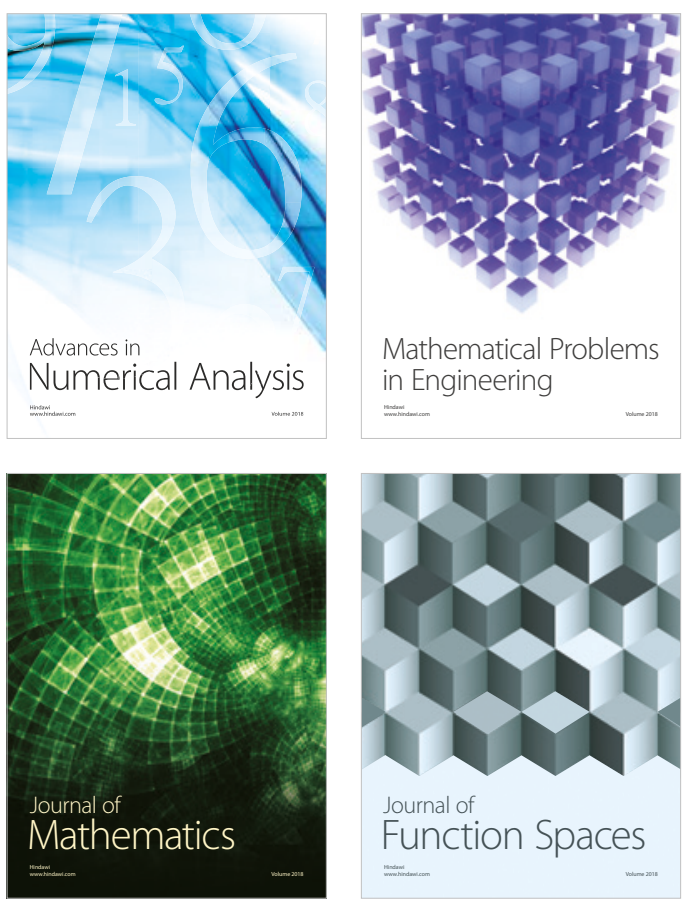

Mathematical Problems in Engineering

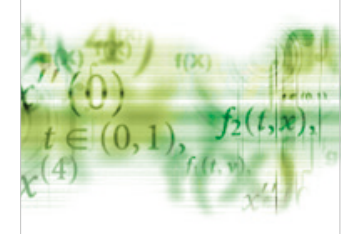

International Journal of

Differential Equations

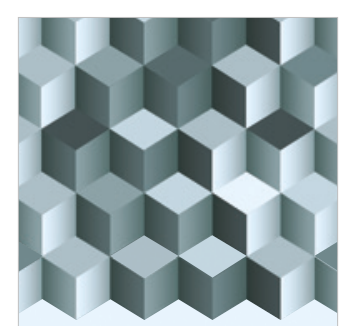

Journal of

Function Spaces

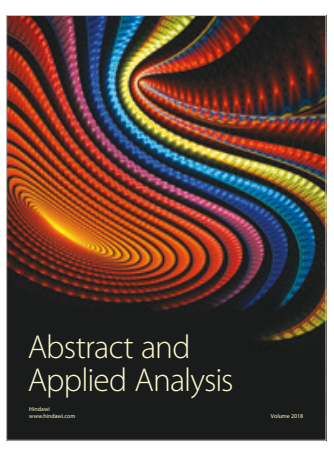

The Scientific

World Journal

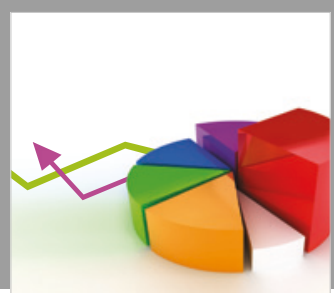

Journal of

Probability and Statistics
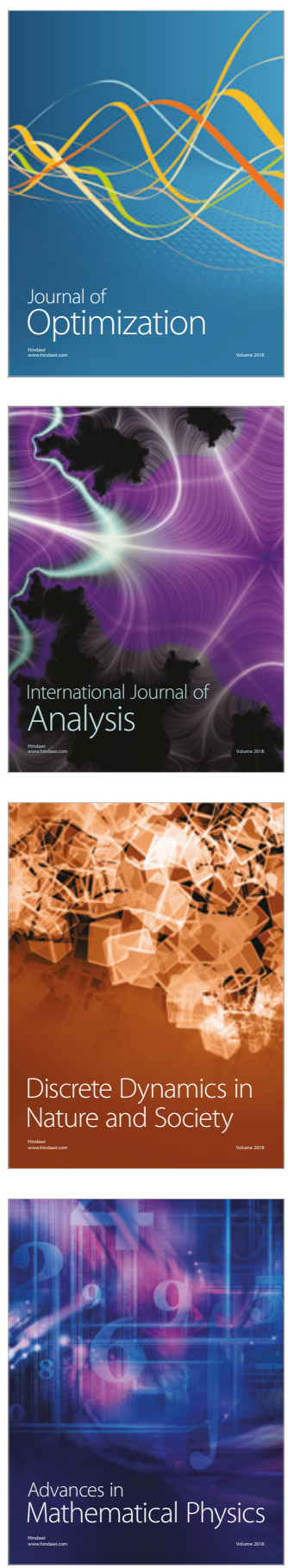